\title{
Fine-scale density wave structure of Saturn's rings: A hydrodynamic theory
}

\author{
E. Griv and M. Gedalin
}

\author{
Department of Physics, Ben-Gurion University of the Negev, PO Box 653, Beer-Sheva 84105, Israel \\ e-mail: griv@bgu.ac.il
}

Received 12 March 2010 / Accepted 13 July 2010

\begin{abstract}
Aims. We examine the linear stability of the Saturnian ring disk of mutually gravitating and physically colliding particles with special emphasis on its fine-scale $\sim 100 \mathrm{~m}$ density wave structure, that is, almost regularly spaced, aligned cylindric density enhancements and optically-thin zones with the width and the spacing between them of roughly several tens particle diameters.

Methods. We analyze the Jeans' instabilities of gravity perturbations (e.g., those produced by a spontaneous disturbance) analytically by using the Navier-Stokes dynamical equations of a compressible fluid. The theory is not restricted by any assumptions about the thickness of the system. We consider a simple model of the system consisting of a three-dimensional ring disk that is weakly inhomogeneous and whose structure is analyzed by making a horizontally local short-wave approximation.

Results. We demonstrate that the disk is probably unstable and that gravity perturbations grow effectively within a few orbital periods. We find that self-gravitation plays a key role in the formation of the fine structure. The predictions of the theory are compared with observations of Saturn's rings by the Cassini spacecraft and are found to be in good agreement. In particular, it appears very likely that some of the quasi-periodic microstructures observed in Saturn's A and B rings - both axisymmetric and nonaxisymmetric ones are manifestations of these effects. We argue that the quasi-periodic density enhancements revealed in Cassini data are flattened structures, with a height to width ratio of about 0.3 . One should analyze high-resolution of the order of $10 \mathrm{~m}$ data acquired for the $\mathrm{A}$ and $\mathrm{B}$ rings (and probably $\mathrm{C}$ ring as well) to confirm this prediction. We also show that the gravitational instability is a potential cluster-forming mechanism leading to the formation of porous 100 -m-diameter moonlets of preferred mass $\sim 10^{7} \mathrm{~g}$ each embedded in the outer A ring, although this has yet to be directly measured.
\end{abstract}

Key words. planets and satellites: general - planets and satellites: rings - planets and satellites: individual: Saturn - instabilities hydrodynamics

\section{Introduction}

The dynamics of highly flattened, rapidly rotating gravitating systems have been studied quite thoroughly. This research has attempted to explain the origin of spiral and ring structures in galaxies, in disks around supermassive black holes, in protostellar and protoplanetary clouds, in Saturn's ring system, in the narrow and widely separated ring system of Uranus, etc. A primary theme has been to analyze the perturbation dynamics of these systems, in both linear and nonlinear regimes. We refer to Lin \& Lau (1979), Shu (1984), Papaloizou \& Lin (1995), Lin \& Papaloizou (1996), Bertin \& Lin (1996), Bertin (2000), and Yuan (2002) for reviews of the theory and its applications. It has been shown that nonuniformly rotating self-gravitating astrophysical disk configurations are highly dynamic and subject to various collective instabilities of gravity perturbations (e.g., those produced by a spontaneous disturbance or, in rare cases, a companion system). This is because their evolution is primarily driven by angular momentum redistribution. The system may then fall toward the lower potential (orbital) energy configuration and use the energy so gained to increase its coarse-grained entropy (Lynden-Bell \& Kalnajs 1972; Griv \& Gedalin 2004; Griv et al. 2008).

The theoretical studies of Maxwell (1859) demonstrated that the rings around Saturn might not be either solid or liquid, but rather a swarm of millions of individual particles rotating in separate concentric orbits at different speeds. A modern very popular model of the particles in Saturn's rings is a smooth ice sphere, whose restitution coefficient is quite high and decreases as the collision velocity increases (e.g., Kerr 1985). Ring particles are in circular orbital motion in Saturn's equatorial plane, at circular velocities $v_{\text {circ }} \sim 10 \mathrm{~km} \mathrm{~s}^{-1}$ and relative (random) velocities $v_{\text {rand }} \lesssim 0.1 \mathrm{~cm} \mathrm{~s}^{-1}$. The Saturnian ring disk consists predominantly of water-ice particles ranging between about $1 \mathrm{~cm}$ and $5 \mathrm{~m}$ in radius $a$ with a distance between them of about a few meters; the bulk of Saturn's ring mass is assumed to reside in individual particles in the 1-3 m diameter range (Shu 1984; Zebker et al. 1985; French \& Nicholson 2000; Cuzzi et al. 2002, 2009; Esposito 2002; Griv \& Gedalin 2003). Numerical simulations have indicated that the larger particles are nearly confined to a monolayer, with the smaller particles filling the spaces between the larger particles (Zebker et al. 1985; Altobelli et al. 2008). The comparison of ground-based visible data with CASSINI CIRS observations also indicated that the A ring is a monolayer (Leyrat et al. 2008a; Ferrari et al. 2009).

VOYAGER flybys of Saturn have revealed that the disk about the planet is not simply divided into several main bands, the classical A-C rings, but that the entire disk assembly is indeed subdivided into a huge number of fine thread-like rings with the appearance of record-grooves (Smith et al. 1981, 1982; Lane et al. 1982; Stone \& Miner 1982; Brahic 2001, Figs. 7-9 therein; Cuzzi et al. 2002, Fig. 2b therein; Esposito 2002, Fig. 5 therein). It is important that the VOYAGER's photopolarimeter PPS data detected some indirect evidence of "finest" structuring in the 
densest central parts of the opaque Saturn's B ring down to the $100 \mathrm{~m}$ length scale (Esposito et al. 1983; Showalter \& Nicholson 1990; Nicholson \& Dones 1991; Horne \& Cuzzi 1996; Esposito 2002, p. 1751). However, below a length scale of a few kilometers, the VOYAGER's PPS data is too noisy to extract information about the structure: the finest structure observed by PPS is accurately descibed by models of statistical noise combined with stochastic variations resulting from large particles or clumps of particles (Showalter \& Nicholson 1990). It was suggested numerically by Salo $(1992,1995)$ and analytically by Griv (1998, 2005a), Griv et al. (2000, 2003a,b), and Griv \& Gedalin (2003, 2005) that far more precise CASSINI spacecraft observations would help us to resolve the question.

The fine-scale of the order of $100 \mathrm{~m}$ or even less quasiperiodic density structure of the Saturnian brightest A and $\mathrm{B}$ rings has indeed been discovered by CASSINI science images (Porco et al. 2005, Figs. 5A and F therein), and then CASsINI UVIS and radio occultations (Colwell et al. 2006, 2007; Thomson et al. 2007). CASSINI VIMS observations (Hedman et al. 2007) and infrared observations of Saturn's rings by CASSINI CIRS (Leyrat et al. 2008a; Ferrari et al. 2009) also detected fine structure in the A ring. Opaque particle clumps called self-gravity wakes are separated by nearly empty, opticallythin gaps. Interestingly, both nonaxisymmetric structures that have a characteristic trailing orientation of $\psi \approx 20^{\circ}$ relative to local direction of orbital motion (Colwell et al. 2006, 2007; Hedman et al. 2007; Leyrat et al. 2008a; Ferrari et al. 2009) and azimuthally symmetric structures with the spatial orientation $-3^{\circ} \leq \psi \leq+3^{\circ}$ (Colwell et al. 2007, p. 141; Thomson et al. 2007, p. L24203) were found. These structures are found to have an average height-to-spacing ratio $H / \lambda=0.2-0.3$ and a width to spacing ratio of $W / \lambda=0.3-0.4$. Gaps between wakes, which are filled with particles, have a low optical depth $\tau=0.1-0.2$. To reiterate, CASSINI results show that in both the A and B rings, axisymmetric microstructure, characterized by a periodic radial variation in optical depth, coexists with nonaxisymmetric microstructure. The spacing between these structures vary from $30 \mathrm{~m}$ to $250 \mathrm{~m}$, depending on the location in the rings. Nicholson et al. (2008) noted that the quasi-periodic microstructure in the inner $\mathrm{B}$ ring appears to be unchanged in amplitude and phase over an interval of 24 years. Both spacecraft missions have shown that these relatively large "irregular variations" in optical depth are not associated with any resonances with known satellites. On a small scale, the fine variations have surprizingly been observed to undergo variation and oscillations with time and ring longitude (Lane et al. 1982; Smith et al. 1982). The latter indicates that the irregular variations are probably wave phenomena, and different instabilities of small-amplitude gravity perturbations may play important roles in ring dynamics. A wavelet analysis of the structure of $\mathrm{A}-\mathrm{C}$ rings has already shown that the rings exhibit various wave perturbations, which weakly interact with each other (Postnikov \& Loskutov 2007). One concludes that at the sub-km level the dense A and B rings are dominated by an elongated, quasi-periodic microstructures also known as self-gravity wakes in the literature (see Nicholson \& Hedman 2010 , for a discussion $)^{1}$.

In our model, Saturn's rings consist of primarily larger than $1 \mathrm{~cm}$ size identical, almost elastically colliding, and gravitating particles. The model formation is thought to start with partially inelastically colliding particle settling onto the central plane of a rotating cloud to form a thin and relatively dense disk around

\footnotetext{
1 We consider the latter name, namely "self-gravity wakes", less than apt and will avoid it here (see also Colwell et al. 2006, for a discussion).
}

the plane. Because of inelastic physical impacts, the disk radiates heat from its surface and, therefore, cools becoming thinner and thinner. The mere existence of flattened rings implies dissipation by means of partially inelastic impacts, which takes some $10^{6} \mathrm{yr}$ to complete. Subsequently, the local gravitational instability causes the disk on attaining a certain critical thickness, which is small in comparison with the outer radius of the system $R$ (and, correspondingly, very low temperature), to disintegrate spontaneously into a number of separate rings and spirals ${ }^{2}$. Thus, we consider the gravitationally unstable rings to be an annular disk with concentric circles and spiral local maxima and minima in both density and brightness. It is natural to assume that the growth rate of the gravity perturbations of an originally weakly unstable cloud was rather low. Therefore, we investigate perturbations that are relatively small compared to equilibrium (though of finite amplitude).

\section{Formulation of the problem}

As for high-temperature plasmas, a system of particles in Saturn's rings exhibits collectively unstable modes of motions. Because of its long-range Newtonian forces, a self-gravitating medium (a particulate "gas", say) possesses collective motions in which all the particles of the system participate. These properties should be manifested in the behavior of small gravity perturbations arising against the equilibrium background. "Collective processes are completely analogous to two-body collisions, except that one particle collides with many which are collected together by some coherent process such as a wave" (Kulsrud 1972, p. 338). Thus, relaxation in particulate systems can occur without ordinary collisions by means of the influence of collective motions of the particulate gas on the particle distribution (Kulsrud 1972; Sagdeev \& Galeev 1969; Galeev \& Sagdeev 1983; Griv et al. 2006b). A cloud of "single charged" particles of Saturn's rings can be unstable against azimuthal and axial perturbations, i.e., in general, inconsistent with a being in thermodynamic equilibrium.

The number of mechanisms producing the ubiquitous finescale structure of Saturn's rings has grown rapidly in the past 25 years. In a review by Griv \& Gedalin (2003), seven mechanisms are listed. However, along with this growth in the number of known mechanisms, there has been a growth in our understanding that a universal mechanism may generate the density structure in all regions of Saturn's main rings. In the present paper of the series, we present the subject of Saturn's rings instabilities in such a way as to emphasize that this universal mechanism exists. We regard this microstructure of rings about Saturn as a wave pattern, which does not remain stationary in a frame of reference rotating around the planet at a proper speed, excited as a result of Jeans' nonresonant (or algebraic) gravitational instability. The nonaxisymmetric wave structure structure rotates uniformly, although the material rotates differentially and the spirals (and rings) consist of different material at different times.

\footnotetext{
2 Destabilizing self-gravity in far more "dangerous" in thin disks than in thick disks. If a rotating disk has a large vertical thickness owing to a high internal temperature, then it is stabilized against all gravitational instabilities. Instabilities arise as the thickness of the layer is reduced (Safronov 1980; Shu 1984). We are interested only in thin astrophysical systems with a ratio of the half-thickness $h$ to the outer radius $R$ much smaller than unity: $h / R \ll 1$. This ratio is characteristic of all spiral galaxies, the gaseous disks around black holes, the planet-forming disks of protostars, and planetary rings. The main Saturn's rings are only about $10 \mathrm{~m}$ thick.
} 
On the other hand, there are regular ringlet complexes in the $\mathrm{A}-\mathrm{C}$ rings connected to resonances with external satellites, including Lindblad horizontal and vertical resonances (Holberg et al. 1982; Lissauer \& Cuzzi 1982). The structures associated with this kind of resonances are directly observed as the socalled spiral or bending wave trains (Shu et al. 1983; Shu 1984; Rosen et al. 1991; Yuan 2002, Fig. 3 therein; Esposito 2002, p. 1752; Porco et al. 2005, Figs. 5G and H therein; Sicardy 2005, p. 463; Tiscareno et al. 2006a, 2007; Colwell et al. 2009). The trains are the resonantly excited density waves that decay as they propagate away from the resonances. Second-order waves were also clearly resolved in the CASSINI data (Tiscareno et al. 2006a). The waves can be used as diagnostics to obtain fundamental physical parameters that characterize the dynamical state of the ring such as mass, thickness, and collision velocities (Shu et al. 1983, 1985; Yuan 2002). Hedman et al. (2009) presented images of resonant structures in Saturn's faint G ring, the inner Roche Division, and the middle D ring. Most of the structures in Saturn's A and B rings, however, do not correspond to resonances with known satellites: wave trains associated with known resonances cover less than $1 \%$ of the radial extent of the $\mathrm{A}$ and B rings (Goldreich \& Tremaine 1982; Horn \& Cuzzi 1996). The study of these regular wave ringlets is beyond the scope of the present paper. We also do not consider few truly isolated ringlets with adjacent empty gaps, located in the low-density $\mathrm{C}$ ring, in the inner $\mathrm{B}$ ring, in the A ring, and in the Cassini Division, resembling those of Uranus (Porco 1990; Esposito 2002, p. 1756). Many of the narrow ringlets with typical widths of a few tens of kilometers and extremely sharp edges are found in the isolated resonance locations of different satellites (Rosen et al. 1991). An external satellite takes angular momentum from the particles of a disk, and the resultant angular momentum transfer can open gaps or terminate rings (e.g., Griv 2007a).

It is convenient to divide instabilities into two broad classes: (a) macroscopic and (b) microscopic. The macroscopic, or hydrodynamic, instabilities imply the displacement of macroscopic portions of rings - all the particles in a given macroscopic volume execute the same average motion. The gasdynamic equations and the continuity equation can be used for the theoretical analysis of this class of instabilities. The microscopic, or kinetic, instabilities can be defined as those for which the differences in the motion of different particles in the same volume are important. The Boltzmann equations are necessary for the analysis of these instabilities. Our aim is to present the linear theory of macroscopic Jeans instabilities of Saturn's rings. The present instability theory assumes that the departure of the system from dynamical equilibrium is infinitesimal and then ask whether this infinitesimal departure grows or decays. Notice that although the linear theory does not establish the amplitude of the perturbations, it does yield values of their dispersion properties and by means of stability criteria it can determine some of the equilibrium parameters of the system under study.

The Jeans instability sets in when the destabilizing effect of the self-gravity in the disk exceeds the combined restoring action of the pressure and Coriolis forces. The wave propagation is a process of rotation as a solid about the center at a fixed phase velocity, despite the general differential rotation of the system; the alternating density enhancements and relatively rarefied zones consist of different material at different times ${ }^{3}$. The classical Jeans instability of gravity disturbances is one of the most

3 If the nonaxisymmetric waves only consisted of the same particles, than these waves would "wrap up" around the planet and essentially smear out of visibility. frequent and important instabilities in the stellar and planetary cosmogony, and galactic dynamics. The term gravitational instability, as introduced by Jeans (1928), deals with the question of whether initial density fluctuations will either be amplified or die down. Jeans instability identifies nonresonant instabilities of gravity fluctuations associated with almost aperiodically growing accumulations of mass, and the dynamics of Jeans perturbations can be characterized as a fluidlike wave-particle interaction. In other words, the instability associated with departures of macroscopic quantities from the dynamical equilibrium is hydrodynamical in nature and has nothing to do with any explicit resonant $\omega=\boldsymbol{k} \cdot \boldsymbol{v}$ effects, where $\omega$ is the oscillation frequency, $\boldsymbol{k}$ is the wavenumber, and $\boldsymbol{v}$ is the particle's velocity. Following Lin \& Shu (e.g., Lin et al. 1969), a relatively simple hydrodynamical model can be used to investigate the instability (Lau \& Bertin 1978; Lin \& Lau 1979; Morozov 1985; Montenegro et al. 1999; Griv 2006). Thus a kinetic description (e.g., Bertin 1980; Griv et al. 2002) yields results that are almost no different from those obtained hydrodynamically. Ginzburg et al. (1972) first examined the possible gravitational instability of Saturn's rings of the type discussed by Lin \& Shu in the context of the formation of spiral arms of normal galaxies.

The difficulties in a satisfactory understanding of the dynamics of particulate systems are due to the well known fact that systems of $N$ gravitationally interacting particles are naturally inhomogeneous, because gravitational forces between gravitational "charges" (which are always "charges of the same sign") are always attractive. The density of the gravitating disk decreases towards its periphery. This inherent inhomogeneity character is the origin of some mathematical problems. For instance, in the case of spiral galaxies one has to deal with complex boundary problems while in a plasma the model of a homogeneous infinitely extended background already allows one to obtain many familiar effects. Following plasma physics, however, the problem may be simplified by considering the so-called WenzelKramer-Brillouin (WKB), or short-wave local approximation. Under the local consideration (in the vicinity of a given point) of perturbations with scales small compared to a characteristic linear dimension $R$ of the system, one may assume parameters of the stationary state equal to its values in a given point. The local approach applied to the gravitational instability and to many types of instabilities in an inhomogeneous self-gravitating systems is valuable, in particular for understanding the physics of the phenomenon considered, since it leads to rather simple analytical results.

In closing a section, one usually chooses a model of Saturn's rings consisting of an infinitesimally thin disk of particles with the surface density equal to the projection of the full mass density on the plane perpendicular to the rotating axis, i.e., a disk, the equilibrium thickness $2 h$ of which is many times smaller than the perturbation radial wavelength $\lambda_{\mathrm{r}}=2 \pi / k_{\mathrm{r}}$, where $k_{\mathrm{r}}$ is the radial wave number. It has been stated that this accuracy is sufficient for the discussion of oscillation modes in Saturn's rings of small but finite disk thickness. As is known, the standard Lin-Shu dispersion relation for planar density waves hypothesized to propagate in galaxies (Lin \& Shu 1966; Lin et al. 1969; Shu 1970) has been slightly improved in either a heuristic manner (Lin \& Shu 1968; see also Toomre 1964; and Safronov 1980) or by introducing an approximate reduction factor providing the correction for finite thickness (Vandervoort 1970). We refer to Shu (1984) for a review of the problem. The method employed by Vandervoort (1970) to solve the collisionless Boltzmann equation when normal to the equatorial plane, $z$-motions are taken into additional account is based on the existence of an 
(a)

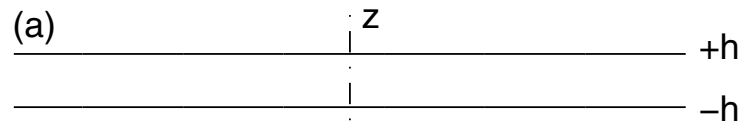

(b)

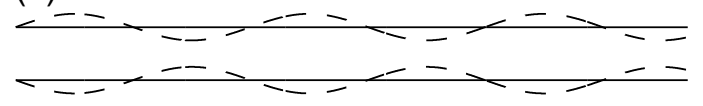

(c)

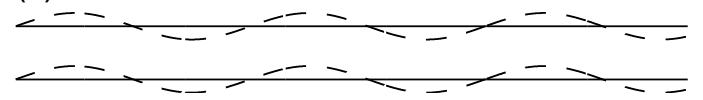

Fig. 1. Sketch of perturbations of a three-dimensional disk. In a) a section of the disk is shown edge-on. In b) a mode of even symmetry with respect to the equatorial plane, or an even-parity Jeans-type perturbation, is shown (the dashed line). In c) a mode of odd symmetry with respect to the equatorial plane, or an odd bending-type perturbation, is illustrated (the dashed line).

adiabatic invariant $J_{z}$, whose approximate constancy characterizes the vertical motion of disk particles. Romeo (1992) already stated that this is a characteristic of only highly flattened, rotationally supported astrophysical disks, where the frequency of the free epicyclic oscillations in the $z$ direction is large compared to the frequency of the epicyclic motion in the symmetry plane, which in turn is generally of the same order as the pattern frequency of spiral waves. We present a more rigorous investigation of the effect of finite thickness (or the particle velocity spread in the $z$ direction) on the propagation of collective oscillations. A different approach from the rest is taken: (1) to study the effect of thickness on even-parity gravity perturbations of the kind studied by Lin \& Shu (Fig. 1b), which do not distort the plane of a disk, by considering the arbitrary but not only highly flattened, rapidly rotating (Vandervoort 1970, p. 94; Shu 1984, p. 557; Romeo 1992, p. 311) system and (2) to treat the oddparity perturbations of the bending type (Fig. 1c), which do distort the disk's plane. The problem is formulated in the same way as in plasma theory (Arsenin 1967; Harris 1968; Alexandrov et al. 1984, p. 313). This approach is introduced here for the first time in an astrophysical context.

\section{Basic equations}

An extended flat Saturnian ring disk of identical, mutually gravitating, and elastically colliding particles orbiting the planet is studied, with $M_{\mathrm{p}}$ being Saturn's mass, $M_{\mathrm{d}}$ being the mass of the disk, and $M_{\mathrm{d}} / M_{\mathrm{p}} \ll 1$. That $h / R$ is small means that the disk considered by us is rather dynamically cold and that the pressure gradient in it is much smaller than both the gravitational and the centrifugal forces. The model assumes ring particles to be confined by a strong Saturnian gravitational field. To formulate the problem, we must include effects of self-gravitation, pressure gradients, and viscosity. The evolution of the system can be described by the basic equations of viscous fluid dynamics, or Navier-Stokes model motion equations

$$
\frac{\partial \boldsymbol{v}}{\partial t}+(\boldsymbol{v} \cdot \boldsymbol{\nabla}) \boldsymbol{v}=-\boldsymbol{\nabla} \Phi-\frac{1}{\rho} \boldsymbol{\nabla} P-\eta \nabla^{2} \boldsymbol{v}+\left(\xi+\frac{\eta}{3}\right) \boldsymbol{\nabla}(\boldsymbol{\nabla} \cdot \boldsymbol{v})
$$

and the continuity equation

$$
\frac{\partial \rho}{\partial t}+\nabla \cdot(\rho \boldsymbol{v})=0
$$

where $\rho(\boldsymbol{r}, t)$ is the volume mass density, $\Phi(\boldsymbol{r}, t)$ is the total gravitational potential (including the planet), such that the gravitational force $\boldsymbol{F}_{\text {grav }}=-\boldsymbol{\nabla} \Phi, P(\boldsymbol{r}, t)$ is the pressure, and $\boldsymbol{v}(\boldsymbol{r}, t)$ is the fluid velocity (Landau \& Lifshitz 1987). Given the geometry of the problem, we adopt cylindrical polar coordinates $(r, \varphi, z)$ centered on the planet and the axis of the disk rotation is along the $z$-axis. Physically in Eq. (1), the terms with $\eta$ and $\xi$ describe the viscosity, and $\eta>0$ and $\xi>0$, respectively, are the velocityindependent coefficients of shear and bulk viscosity. The viscosity being driven by collisions between particles. The compressibility of the gas assumes $\xi \neq 0$. The viscosity is assumed to be independent of density and position. In hydrodynamics, the viscosity coefficients $\eta$ and $\xi$ are considered to be known. As is known, Eq. (1) is simply a statement of Newton's second law: the left-hand side is the acceleration and the right-hand side is the various forces acting on the fluid. Among these forces, we have gravity $\propto \Phi$, pressure forces $\propto P$, and viscous forces $\propto \eta, \xi$. The equation of state is isothermal

$P=c_{\mathrm{s}}^{2} \rho$,

and $c_{\mathrm{S}}$ means a characteristic speed of sound in the medium that can be represented by

$c_{\mathrm{s}}^{2}=(\partial P / \partial \rho)_{\text {adiabatic }}$.

Thus, at the outset we adopt an isothermal fluid model for Saturn's rings, identifying the velocity dispersion of identical particles in the rings with the sound speed in our model. In a self-consistent problem, Eqs. (1)-(3) must be solved simultaneously with the Poisson equation

$\frac{\partial^{2} \Phi}{\partial r^{2}}+\frac{1}{r} \frac{\partial \Phi}{\partial r}+\frac{1}{r^{2}} \frac{\partial^{2} \Phi}{\partial \varphi^{2}}+\frac{\partial^{2} \Phi}{\partial z^{2}}=4 \pi G \rho$

Owing to their velocity dispersion, ring particles experience collisions. In its simplest form, the nature of the disk viscosity is physical collisions and the frequency of collisions is obviously $v_{\text {coll }} \approx n\langle s v\rangle$, where $n$ is the number density of particles, $\langle\cdots\rangle$ denotes the average over particles of all random velocities $v$ in a Maxwellian-like distribution, and the particles are imagined to be identical spheres of cross-sectional area $s$. Some relations exist between optical depth, ring thickness, particle density, and other quantities that define the physical state of a planetary ring. Thus, for the thin disk the optical depth is approximately equal to the ratio of the collision frequency to the orbital frequency $\Omega$

$\tau \approx \frac{v_{\text {coll }}}{\Omega}$

(Jeffreys 1947; Cook \& Franklin 1964; Goldreich \& Tremaine 1982; Bridges et al. 1984; Stewart et al. 1984; Araki 1991). In the three-dimensional disk, the characteristic frequency of collisions is indeed

$v_{\text {coll }} \approx\langle v\rangle \sigma_{\text {coll }} n$

where $\sigma_{\text {coll }}$ is now the cross-section for collisions. In the particular case where the two colliding particles are the same with a radius $a$ we obtain the obvious result $\sigma_{\text {coll }} \approx 4 \pi a^{2}$. If we use the condition of equilibrium along the $z$-coordinate in the disk in the field of a large central mass, $h \approx c_{\mathrm{s}} / \Omega$, and introduce the optical depth $\tau$ of the disk in the form (Goldreich \& Tremaine 1982)

$\tau \approx 4 \pi a^{2} n h$

(bearing in mind that $\sqrt{\left\langle v^{2}\right\rangle} \approx c_{\mathrm{s}}$ and $h$ is the semi-thickness of the disk), from Eqs. (6) and (7) we can finally write the expression for $\tau$ in the form of Eq. (5). This is the fundamental 
result that was rediscovered by Shu \& Stewart (1985), employing a Krook approximation to the collision integral. In the following, we argue that fine $\sim 100 \mathrm{~m}$ structures could be primarily produced by the classical Jeans instability in low and moderately high optical depth regions of the system with $\tau<1-2$, that is, in regions of the system with relatively rare collisions between particles, $v_{\text {coll }} \lessgtr \Omega$. These regions can be found in the $\mathrm{C}$ ring, where the average optical depth $\tau<0.1$, in the main portions of the densest B ring, where $\tau=0.5-1.5$, and in the A ring (including the Encke gap), where $\tau \leq 0.5$ (Nicholson et al. 2000; Esposito 2002, Fig. 3 therein). Thus, in the main portion of Saturn's rings the collision frequency of the ring particles is of the order of the orbital frequency or even less (Goldreich \& Tremaine 1982, p. 265; Shu \& Stewart 1985; Yuan 2002, p. 129). We conclude that calculations infer a typical rate of $\lesssim(1-10)$ collisions per 10 orbits, or $\sim 100-200 \mathrm{~h}$, respectively. According to Murray et al. (2008), "the F ring region of Saturn's rings is perhaps the only location in the Solar System where large-scale collisional processes are occurring on an almost daily basis".

In Appendix A below, we show that the system of Eqs. (1)-(4) admits a solution in the form of self-gravity density waves, which were first studied by Lin \& Shu (Lin \& Shu 1966, 1968; Lin et al. 1969; Shu 1970; Lin \& Lau 1979) on larger scales as they exist in galactic disks. These fully selfconsistent Lin-Shu density waves (normal modes) are not to be confused, of course, with Julian \& Toomre's (1966) perturbed or "forced" density wave proposal explored by Colombo et al. (1976), Franklin \& Colombo (1978), Lumme \& Irvine (1979), and Karttunen (1983). Julian \& Toomre (1966) worked out the response of a disk - a calculation of the polarization cloud - when forced by an orbiting mass or clump, such as a giant molecular cloud in spiral galaxies ${ }^{4}$. Accordingly, the integrated mass of the polarization cloud ("wake") may be several times the mass of the original clump (Julian \& Toomre 1966, Fig. 7 therein). According to Colombo et al. (1976), in Saturn's rings "large particles will force intense trailing density wakes" and this Julian-Toomre-type mechamism for producing the azimuthal brightness variation "requires the rings to contain particles whose radii are considerably larger than average". In the spirit of Julian \& Toomre (1966), to calculate the response of a disk to a point mass orbiting within it, one needs to replace the potential $\Phi$ in Eqs. (1)-(4) above by $\Phi+\Phi_{\text {imp }}$, where $\Phi_{\text {imp }}$ is the imposed potential (Julian \& Toomre 1966, p. 811). The results following from Eqs. (1)-(4) obviously critically depend on $\Phi_{\text {imp }}$; the polarization cloud vanishes if $\Phi_{\text {imp }} \rightarrow 0$. Unlike Julian \& Toomre (1966), we develop the self-consistent theory of real instabilities of small spontaneous disturbances (the external potential $\Phi_{\text {imp }}=0$ ), which develop effectively from noise to observable amplitudes in some $20 \mathrm{~h}$ in the main part of the system under study. As for us, Julian \& Toomre's (1966) "forced" instability of a system of almost identical particles cannot serve as the generator of quasi-regular spiral (and ring) density waves with a characteristic pitch angle $\psi_{\text {crit }} \approx 20^{\circ}$ in Saturn's rings ${ }^{5}$. The Lin-Shu-type density wave conception of microstructure adopted in the present paper is less restrictive than the Julian-Toomre one. We suggest that the preferred orientation of self-excited gravitationally unstable density waves

\footnotetext{
${ }^{4}$ Whether these Julian-Toomre-type forced density waves can be excited in astrophysical disks remains controversial (e.g., Sellwood \& Lin 1989, p. 992; Sellwood \& Kahn 1991, p. 278).

5 Salo (1992, 1995) and others (e.g., Griv 2005a) already demonstrated the formation of fine-scale structures in simplified local $N$-body simulations of planetary rings of identical particles.
}

(Lin-Shu-type "free" density waves) produces brightness azimuthal variations that have been observed (see, e.g., Leyrat et al. 2008a,b, for an explanation).

\subsection{Dynamical equilibrium}

We now assume that $M_{\mathrm{d}} / M_{\mathrm{p}} \rightarrow 0$. Let us consider a free particle orbiting in a circle of radius $r$ with angular speed $\Omega(r)$ in the equatorial plane $z=0$ of the non-spherical planet with an associated gravitational potential $\Phi_{\mathrm{p}}(r, z)$. The planar equilibrium of a system is expressed by the condition

$$
r \Omega^{2}=\left.\frac{\partial \Phi_{\mathrm{p}}}{\partial r}\right|_{z=0} .
$$

If this test particle is displaced by an arbitrary small amount, it will oscillate freely in the horizontal and vertical directions about the reference circular orbit with epicyclic frequency $\kappa(r)$ and vertical frequency $\mu(r)$ given by Lindblad's theory of epicyclic motion (Shu 1984; Borderies \& Longaretti 1994),

$$
\begin{aligned}
& \kappa^{2}(r)=r^{-2} \frac{\mathrm{d}}{\mathrm{d} r}\left[\left(r^{2} \Omega\right)^{2}\right], \\
& \mu^{2}(r)=\left.\frac{\partial^{2} \Phi_{\mathrm{p}}}{\partial z^{2}}\right|_{z=0} .
\end{aligned}
$$

In the epicyclic approximation, the motion of a particle is represented as in epicyclic motion along the small ellipse (epicycle) with a simultaneous circulation of the epicenter about the planetary center. Of course, the epicyclic approximation may be applied only when the true particle motion is nearly circular, as in planetary rings, and the collision frequency is small in comparison with $\kappa \approx \Omega \approx \mu$. It follows from Eq. (9) that a nearly circular orbit is stable in the equatorial plane of the disk if $\kappa^{2}>0$; in other words, if $\Phi_{\mathrm{p}}$ tends to $-\infty$ as const $/ r^{n}$ with $n \leq 2$. It is clear, however, that the stability of the individual particle orbits will not guarantee the stability of an actual system $\left(M_{\mathrm{d}} / M_{\mathrm{p}} \neq 0\right)$ against collective oscillations when in addition the time-dependent perturbation of the basic total equilibrium potential is taken into account: the problem may be resolved only by considering the self-consistent system.

The unperturbed disk is assumed to have no motion except for rotation. The present theory suggests some perturbed radial, azimuthal, and vertical motions of the fluid element distributed in the form of a spiral-like flow field, which is a small correction to the basic circular, equilibrium motion. In a plane perpendicular to the rotation axis, the equilibrium motion is described by the equation

$$
r \Omega^{2}=\frac{\partial \Phi_{0}}{\partial r}+\frac{c_{\perp}^{2}}{\rho_{0}} \frac{\partial \rho_{0}}{\partial r},
$$

where $\Phi_{0}$ is the equilibrium potential, $c_{\perp}$ is the speed of sound in the $z=0$ equatorial plane, and the term $\propto c_{\perp}^{2}$ is a small correction motion. Planar equilibrium is therefore established in a simple manner in such a disk, i.e., it is governed mainly by the balance between the centrifugal and gravitational forces. The unperturbed disk has velocity $\boldsymbol{v}_{0}=(0, r \Omega, 0)$, where the angular rotational velocity $\Omega=\Omega(r)$ is taken to be a function of $r$ alone. The equation of hydrostatic equilibrium along the $z$ coordinate (for $z \ll r$ ) is obviously

$$
\frac{\partial \Phi_{0}}{\partial z}+\frac{c_{z}^{2}}{\rho_{0}} \frac{\partial \rho_{0}}{\partial z}=0,
$$

where $c_{z}$ is the sound speed along the normal to the plane direction. By considering the geometrically thin disk, $2 h \ll R$, 

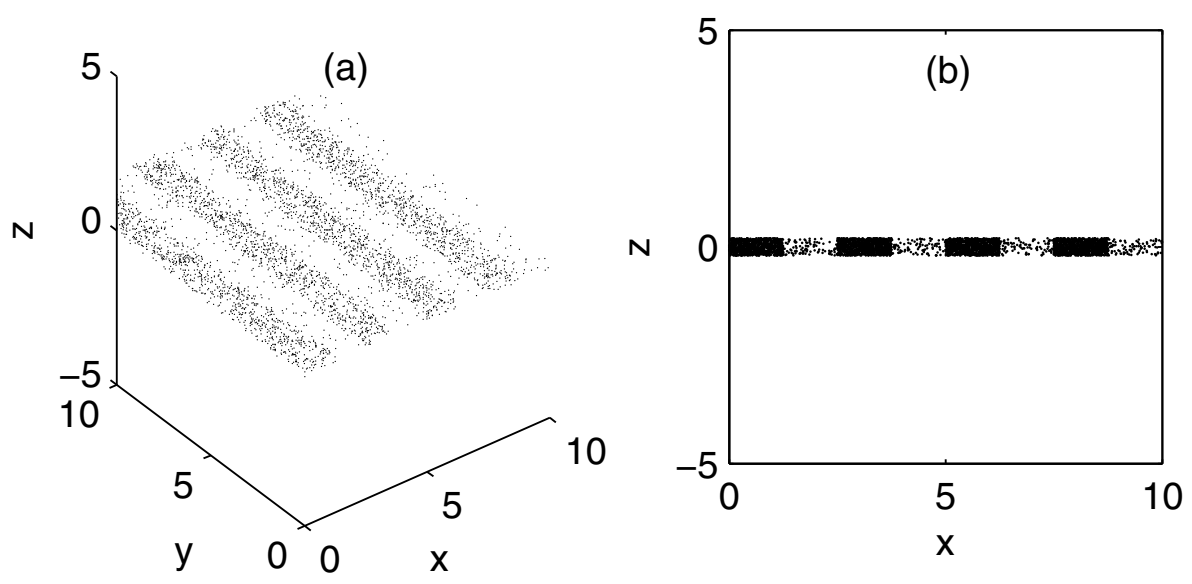

Fig. 2. Schematic model of the fine-scale density wave structure in Saturn's rings. Selfgravity density waves, which were first studied by Lin \& Shu (Lin et al. 1969; Shu 1970), manifest themselves as evenly spaced elongated clusters of ring particles. Shown are both a three-dimensional distribution of particles a) and a distribution of particles in the $(x, z)$-plane b). where $2 h$ is the effective thickness, in Eq. (11) one can expand $\partial \Phi_{0} / \partial z$ about the orbit plane as

$\frac{\partial \Phi_{0}}{\partial z}=\mu^{2} z$

and $\mu^{2}=\left.\left(\partial^{2} \Phi_{0} / \partial z^{2}\right)\right|_{z=0}$ is the frequency of natural vertical oscillations ${ }^{6}$. Equations (11) and (12) then imply that

$\rho_{0}(r, z)=\rho_{0}(r, 0) \exp \left(-\frac{\mu^{2} z^{2}}{2 c_{z}^{2}}\right)$,

where $\rho_{0}(r, 0)$ is the density on the equatorial plane. Obviously, the perturbed density, $\rho_{1}$ and $\left|\rho_{1}\right| \ll \rho_{0}$, in a realistic Saturn's ring disk will have a similar vertical profile, and so will the perturbed potential $\Phi_{1}$. Notice that concerning in particular the vertical structure, detailed theoretical studies including both impacts and self-gravity have been performed, for example by Mosqueira (1996).

Since realistic three-dimensional Saturn's rings are extremely difficult to treat, we consider an idealized model. First, in Eq. (13) for a weakly spatially inhomogeneous disk we consider values of $\rho_{0}$ and $P_{0}$ at $r=r_{0}$ (the local approximation); their distributions are assumed to be symmetric with respect to the $z=0$ plane. Second, in a first step of our study and in the spirit of Mark (1971), Kulsrud et al. (1971), and Bertin \& Casertano (1982), the density in the equilibrium state, along the $z$ coordinate is regarded as uniform between two boundaries $-h$ and $+h$, with a vacuum exterior (Fig. 1a). In other words, following these authors, we consider here a slab equilibrium with plane-parallel symmetry that is homogeneous in the vertical, $z$-direction. Furthermore, we restrict ourselves to consideration of only Lin-Shu unforced density waves, or "heavy sound," which are nothing but longitudinal compression waves in which the self-gravitation of the fluctuations in density is taken into account. These modes have wave vectors perpendicular to the axis of rotation (Fig. 1b). $\mathrm{N}$-body experiments have already identified these longitudinal collective motions for a system of mutually gravitating particles in computer models of Saturn's rings, that is, the particle motion is restricted to be almost parallel to the equatorial plane of the system (Griv 2005a). The waves compress existing material and cause both spiral and clump ("moonlets") formation. The modes of odd symmetry with respect to the equatorial plane (Fig. 1c) deserve a separate investigation. Thus,

\footnotetext{
6 The disk is geometrically thin if $2 h \ll r$, which from the equation $h=c_{z} / \mu$, where $c_{z} \sim c_{\perp}$ and $\mu \approx \Omega$, is equivalent to the disk being dynamically cold, $c_{\perp} \ll r \Omega$, i.e., the sound speed of the ring disk being much less than the orbital speed $r \Omega$.
}

under the action of the gravitational field of the planet, the particles move along almost closed orbits. It is this equilibrium model of the Saturnian ring disk that is examined for stability in the present investigation. The schematic model of self-gravity Jeans-unstable density waves we investigate is shown in Fig. 2.

\section{Astronomical implications}

In Appendix A, according to the local approximation of the WKB method it is shown that Saturn's ring disk rotating differentially could be unstable because of Jeans' gravitational instability. To suppress Jeans instabilities, a generalized local stability criterion must be satisfied, which indicates that in a differentially rotating disk, nonaxisymmetric perturbations are more unstable than axisymmetric ones. Equation (A.31) indicates the stabilizing influence of the finite thickness of a disk, which causes a shift in the threshold of instability toward a longer wavelength (and larger wavelength will include more mass). The etiology of this fluidlike instability is the nonresonant interaction of particles with Jeans-unstable gravity perturbations in a disk. It is similar to the instability of the bunching type in plasmas, e.g., pinch instabilities or a firehose instability. The nonresonant gravitational instability does not depend on the behavior of the particle distribution function in the neighborhood of a particular speed, but the determining factors of the instability are macroscopic parameters such as the velocity dispersion, mean density, and angular velocity of regular rotation. We now proceed to discuss the extent to which our results on the disk's stability can have a bearing on observable Saturn's rings.

\subsection{Stabilizing effect of the finite thickness}

The Saturnian ring disk is regarded as being at the threshold of instability. At the limit of gravitational stability $h \approx c_{\mathrm{T}} / \kappa$ and we assume that the viscosity to have no essential effect on the criterion of the gravitational instability. Substituting this expression for $h$ into Eq. (A.34), we estimate the stabilizing factor introduced by the finite thickness $\left(1-\kappa h / 2 c_{\mathrm{T}}\right) \approx 0.5$. Morozov $(1980,1981)$ took into account the additional weak destabilizing effect of a density inhomogeneity and a radial gradient in velocity dispersion. The result is that the stabilizing effect introduced by the finite thickness and the destabilizing effect introduced by the inhomogeneity and the velocity gradient practically cancel each other out, at least in the local stellar disk of our own Galaxy. In practice, one can neglect all these corrections. We expect therefore that in Saturn's rings $c_{\perp} \approx(2 \Omega / \kappa) c_{\mathrm{T}} \approx 2 c_{\mathrm{T}}$ (or Toomre's stability parameter $Q=c_{\perp} / c_{\mathrm{T}} \approx 2$ ). Interestingly, 
about the same value of $Q \sim 2$ bring the observations of Saturn's rings (Lane et al. 1982, p. 543).

\subsection{Fine-scale structure}

Thus, the modified Safronov-Toomre stability criterion given by Eq. (A.34) is deduced, according to which the system is violently Jeans-unstable against the growth of nonaxisymmetric perturbations, unless the sound speed is at least a factor of two (Toomre's stability parameter $Q \gtrsim 2$ ) higher that required by the usual Safronov-Toomre stability criteria $c_{\mathrm{T}}$ for axisymmetric perturbations. The strongest growth occurs on the scale of $\lambda_{\text {crit }} \sim$ $2 c_{\perp}^{2} / G \sigma_{0}$. In low and moderately high optical depth regions of the $\mathrm{A}$ and $\mathrm{B}$ rings, $c_{\perp} \sim 0.1 \mathrm{~cm} \mathrm{~s}^{-1}$ and $\sigma_{0}=30-100 \mathrm{~g} \mathrm{~cm}^{-2}$, thus $\lambda_{\text {crit }}=30-100 \mathrm{~m}$, that is, the wavelength assumes a value of roughly several tens of particle diameters. These values of $Q \sim 2$ and $\lambda_{\text {crit }} \lesssim 100 \mathrm{~m}$ predicted in our analysis are close to Salo (1992, 1995), Richardson (1994), Osterbart \& Willerding (1995), Griv (1998, 2005a), Daisaka \& Ida (1999), Ohtsuki \& Emori (2000), Griv \& Gedalin (2005), and Griv et al. (2006a) numerical results. These fine waves were indeed found in data obtained by the CASSINI spacecraft. The gravitation plays a key role in the formation of fine-scale structure.

Once a disk is violently unstable (the Safronov-Toomre criterion $Q<1$ ), both axisymmetric and nonaxisymmetric modes of comparable wavelength should grow at comparable rates and the medium fragments into patches of particles with wavelength $\lambda=\lambda_{\text {crit }}$ in which the pressure cannot prevent the fragmentational collapse. The geometric model of self-gravity spiral density waves in Saturn's rings we study is shown in Fig. 2. In the lowest approximation of the theory, the density waves are regularly spaced, aligned three-dimensional clusters of ring particles (Fig. 2a). At the limit of the instability $\left(c_{\perp} \approx \pi G \sigma_{0} / \kappa\right)$, the physical scale of the waves in the equatorial $z=0$ plane is predicted to be of the order of

$\lambda_{\text {crit }}=\lambda_{\text {jeans }} \approx \frac{2 c_{\perp}^{2}}{G \sigma_{0}} \approx 4 \pi h$.

We conclude that the most unstable modes of the differentially rotating disk have wavelengths of some $2 \pi$ the thickness $2 h$ of the system. As we already remarked, CASsINI's observations indeed revealed a similar $100 \mathrm{~m}$ quasi-periodic fine structure of Saturn's rings (e.g., Porco et al. 2005). We conclude that the critical wavelength $\lambda_{\text {crit }} \lesssim 100 \mathrm{~m}$ does agree with the width of spirals (and rings) observed in Saturn's A and B rings. It is natural to attribute the observed microstructure to the Jeans instability of spontaneous gravity perturbations so far discussed in this paper.

As for the present study, the Jeans-unstable spiral density waves also cause the ring A's quadrupole azimuthal brightness asymmetry detected first by Camichel (1958) and then by Lumme \& Irvine (1976), Lumme et al. (1977), Thompson et al. (1981), Franklin et al. (1987), Dones et al. (1993), Dunn et al. (2004), and others (see Griv \& Gedalin 2003; and Porco et al. 2008, for a discussion). The physics underlying these selfgravity waves is essentially the same as the "density wave structure" which was studied in the context of galactic disks by Lin \& Shu (1966), Lin et al. (1969), Shu (1970), Lau \& Bertin (1978), Lin \& Lau (1979), Bertin (1980), Morozov (1980), Montenegro et al. (1999), Griv et al. (2002), and others.

\subsection{Formation of clumps}

Thus, if the disk is thin, $c_{\perp} \ll r \Omega$, and dynamically cold, that is, $c_{\perp}<c_{\mathrm{T}}$ (Toomre's stability parameter $Q<1$ ), then this model will be gravitationally unstable to both axisymmetric and nonaxisymmetric perturbations, and should almost instantaneously (see below for a time estimate) take on the form of a cartwheel, that is, a structure of spirals and rings (Fig. 3a). One understands, however, that in the nonlinear stage of evolution there will be some exchange of energy and angular momentum between the axisymmetric and nonaxisymmetric modes that will give rise to a pattern far more complex than the cartwheel shown in Fig. 3a. The real spiral pattern is unlikely to be as regular as illustrated in Fig. 3a. It probably resembles the spiral pattern of ragged, multiarmed Sc galaxies (see Griv 2005b, for a discussion). The ragged, "irregular" structure formation process in planetary rings was demonstrated in the simulations carried out by Salo (1992, 1995) and others (e.g., Daisaka \& Ida 1999; Griv 2005a; Griv \& Gedalin 2005) and observed in CASSINI experiments (e.g., Colwell et al. 2007, p. 142, "the sheets are loosely organized into the trailing spiral density enhancements"). CASSINI observations indicated that axisymmetric periodic microstructure in Saturn's rings coexists with nonaxisymmetric structure (Colwell 2006, 2007; Thomson et al. 2007). Clearly, in this case of both radial and spiral excitation, the distribution of the surface density along the spiral arms is not uniform, but describes a sequence of maxima, that might be identified with forming embedded clusters of particles, resembling a "beads on a string" structure. We argue that the gravitationally bound clusters of particles appear at the intersections of the rings and spiral arms as seen in Fig. $3 \mathrm{a}^{7}$. This disk should break up into discrete, gravitationally confined, and porous blobs of matter ("clumpy moons") of preferred mass

$M_{\text {frag }} \sim \lambda_{\text {crit }}^{2} \sigma_{0} \sim \frac{4 c_{\perp}^{4}}{G^{2} \sigma_{0}}$

and diameters

$D_{\text {frag }} \sim 100 \mathrm{~m}$

distributed in spirals around the spin axis (Fig. 4). In Saturn's rings, the Safronov-Toomre sound speed $c_{\mathrm{T}} \sim 0.1 \mathrm{~cm} \mathrm{~s}^{-1}$ and therefore

$M_{\text {frag }} \sim 10^{7} \mathrm{~g}$.

These values for the ring disk is based on a surface density of $50 \mathrm{~g} \mathrm{~cm}^{-2}$ and an angular speed of $2 \times 10^{-4} \mathrm{~s}^{-1}$ (Nicholson et al. 2005). The clump formation process in ring systems was convinsingly demonstrated in the simulations carried out by Salo (1995, Sect. 7 therein) and Karjalainen \& Salo (2004).

We conclude that the Safronov-Toomre unstable modes might be a potential cluster-forming mechanism (cf. Snytnikov et al. 2004, Figs. 2 and 4 therein; Griv 2005b, Fig. 2 therein). Since the intersections are spaced $\sim \lambda_{\text {crit }}$ apart, it would produce some regularity in the distance intervals between the moon-clusters. Interestingly, low values of thermal inertias of $\mathrm{B}$ and $\mathrm{C}$ ring particles derived from infrared observations of Saturn's rings might be characteristic of very porous particle aggregates (Ferrari et al. 2005). Tiscareno et al. (2006b, 2008, 2010) and Sremčević et al. (2007) presented an extensive

\footnotetext{
7 The latter is an important step towards an understanding of a main question of protoplanetary disk evolution, as well as the evolutionary processes in galactic disks: what kind of evolutionary processes lead to the formation of moons, planets, and stars in a different astrophysical disk system? Maoz (1995) already explained the apparent clustering of the high-velocity emission sources into several distinct clumps near the center of the galaxy NGC 4258 at the intersections of the spiral arms and the rings.
} 

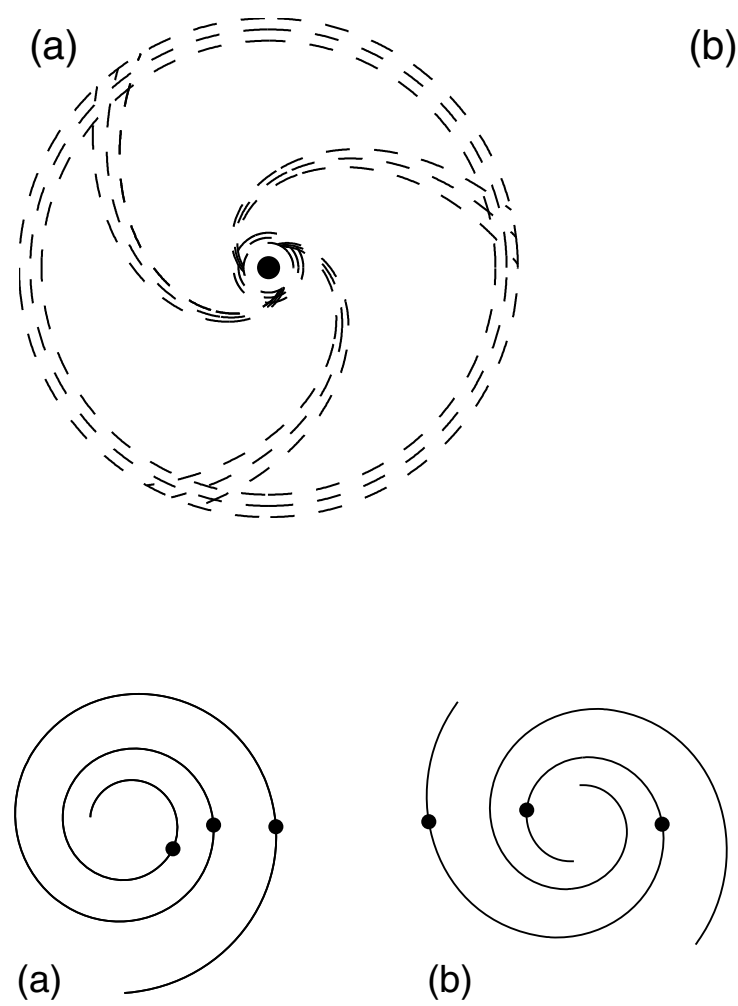

(b) (b)

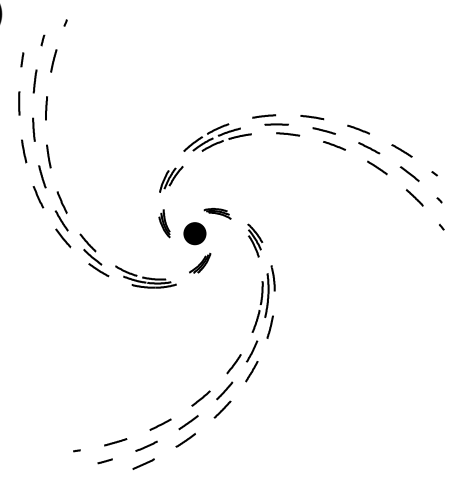

Fig. 3. Schematic model of a Jeans-unstable disk: a) the Safronov-Toomre unstable disk ( $c_{\perp}<c_{\mathrm{T}}$, or $Q<1$, respectively) and b) the Safronov-Toomre stable disk $(Q \geq 1$ but $Q \lessgtr$ $2 \Omega / \kappa$, and in Saturn's rings $2 \Omega / \kappa \approx 2$ ). In the Lin-Shu density wave proposal explored in our theory, the disk particles travel almost freely through the density pattern, so any individual spiral arm (and ring) does not always consist of the same particles. Spirals rotate rigidly with a single fixed pattern speed $\Omega_{\mathrm{p}}$ and therefore do not wind up.

Fig. 4. a) Gravitationally unstable density waves with $m=1 \mathrm{arm}$ in the $(r, \varphi)$-plane, b) density waves with $m=2$ arms, and c) density waves with $m=3$ arms. The filled circles represent the maxima of the perturbed density of Jeans waves, which are unstable to both axisymmetric and nonaxisymmetric gravity perturbations. The distribution of the surface density along the spiral arms is not uniform, but describes a sequence of maxima, that might be identified with forming embedded complexes of particles of a mass of $M_{\text {frag }}$ each.

data set of localized features - "propellers" - from CASSINI images of Saturn's A ring that may be interpreted as signatures of small moonlets embedded within the ring, with diameters between 40 and $500 \mathrm{~m}$ (cf. estimates (15) and (16)). It was noted in particular that the lack of significant brightening at high phase angle indicates that these bodies are likely composed primarily of macroscopic particles, rather than dust $^{8}$. We strongly believe that the clumping Safronov-Toomre instability leads to formation of porous aggregations of multiple smaller objects (moonlets) with diameters $\approx 100-200 \mathrm{~m}$ embedded in broad Saturn's rings?.

\subsection{Nonlinear evolution}

The nonlinear interaction of particles with almost aperiodically growing Jeans-unstable density waves increases the random velocities of particles (via the so-called nonresonant dynamical heating) on a short timescale of only 2-3 disk orbital revolutions (Griv \& Gedalin 2003; Griv et al. 2003a,b). The latter leads to an eventual stabilization (Eq. (A.34)), unless some effective cooling mechanism of the reconstruction of the wave structure exists. It is again suggested that dissipative (inelastic) impacts between particles provide such a cooling mechanism, reducing the

\footnotetext{
8 A close-up of Saturn's rings reveals many bright streaks aligned with the orbital direction of the rings. These objects are the propellershaped features first captured in CASSINI images during the spacecraft's 2004 orbital insertion manoeuvre. Propeller-shaped disturbances occur in Saturn's rings as a result of small moonlets embedded in the rings (Tiscareno et al. 2006). The propellers are most abundant in a $3000 \mathrm{~km}$-wide belt in the mid-A ring, about $130000 \mathrm{~km}$ from Saturn's center (Sremčević et al. 2007). It is estimated that the A ring contains thousands of these objects. Tiscareno et al. (2008) presented a detailed analysis of this population.

9 Note that in the context of protoplanetary disks Toomre's stability parameter $Q<1$ is sufficient to create point masses (giant planets) by disk instability (Boss 2005, 2007).
}

magnitude of the relative velocity of particles, and thus reducing the random velocity spread. In particular we note that the particles constituting Saturn's rings collide experiencing a subsequent loss of mechanical energy. This mechanical energy may be converted into thermal energy, and is essentially lost. The latter leads to some "cooling" of the system, and thus leads to a recurrent instability cycle (Griv \& Gedalin 2003). We argue that both the Saturnian main rings and their irregular fine-scale structure (being recurrently created and destroyed on the timescale of an order of Keplerian period $\sim 20 \mathrm{~h}$ ) are likely not much younger than the solar system (Griv \& Gedalin 2006). Observations of the Saturnian ring system during approach and orbital insertion, with CASSINI's visual and infrared mapping spectrometer, have already shown that Saturn's rings have changed little in their radial structure since the VOYAGER flybys in the early 1980s (Brown et al. 2006).

\section{Discussion}

For the first time in planetary ring dynamics, this paper has examined analytically the stability of three-dimensional Saturn's rings using a hydrodynamic approximation to particle dynamics. The linear analysis is restricted to odd-parity Jeans-type collective modes of oscillations - the Lin-Shu-type compression density waves - for which the vertical velocity $v_{z}(z)=-v_{z}(-z)$ and, in particular $v_{z}(z=0)=0$. It is assumed that Jeans' instability of small-amplitude gravity disturbances is the preferred mechanism to explain both the axisymmetric and the nonaxisymmetric structures present in the $\mathrm{A}$ and $\mathrm{B}$ rings (and probably in the $\mathrm{C}$ ring). The effect on the Saturnian ring disk structure by the dissipative effects (via interparticle collisions) seems likely to play a much smaller role. By assuming that equilibrium rotation and density vary over a much larger spatial scale than the mode wavelength, we found that the most important Jeans' gravitational instability in the Saturnian ring disk is a local instability, i.e., normal modes 
are driven unstable by the local value of the rotational flow shear and the disk gravity.

In previous sections, we have presented some arguments in favour of wave nature of the fine-scale $\sim 100 \mathrm{~m}$ quasi-periodic structure in Saturn's main rings, which is nothing else but a free spiral density wave. From the well-developed theory of galactic spiral density waves, a free density wave is known to rotate in a rigid-body manner and to not be affected by differential rotation of the ring disk.

Thus, one can attribute the fine-scale structure observed in Saturn's A and B rings in CASSINI data to the development of free Lin-Shu density waves developing in the plane of the system. To repeat ourselves, these fully self-consistent density waves, which are an intrinsic property of a gravitationally unstable disk, are not to be confused with Julian \& Toomre's (1966) "forced" density wave proposal explored by Colombo et al. (1976), Franklin \& Colombo (1978), Lumme \& Irvine (1979), and Karttunen (1983). In the spirit of Lin \& Shu (1966), Lin et al. (1969), Shu (1970), Ginzburg et al. (1972), Lau \& Bertin (1978), Lin \& Lau (1979), Bertin (1980), Morozov (1980), Griv et al. (1999, 2002), Montenegro et al. (1999), Griv \& Gedalin (2003), and others, we develop instead the self-consistent theory of real instabilities of small spontaneous disturbances, which grow and propagate effectively in the main part of the system. In our model, the disk particles travel almost freely through the density pattern, so any individual spiral arm (and ring) is not always composed of the same particles; spirals rotate rigidly with a single fixed pattern speed $\Omega_{\mathrm{p}}=\mathfrak{R} \omega_{*} / \mathrm{m}$, hence do not wind up. In sharp contrast to the Julian-Toomre-type (Julian \& Toomre 1966) mechanism for producing the azimuthal (and radial) density variations, the model advocated in this paper does not "require the rings to contain particles whose radii are considerably larger than average" (Colombo et al. 1976). At the limit of gravitational stability, the physical scale of these longitudinal waves (the width and separation of the waves) $\lambda_{\text {crit }}=$ $\lambda_{\text {jeans }} \approx 4 \pi h$ (Eq. (14)) and the disk's thickness is $2 h$. In Saturn's rings, $\lambda_{\text {jeans }}=30-150 \mathrm{~m}$. One concludes that the self-gravity density enhancements as shown in Fig. 2 are flattened structures, with the width and the spacing between them of the order of ten particle diameters and with height/width ratio of about $2 h / \lambda_{\text {jeans }} \lesssim 0.3$. To reiterate, the self-gravity structures are flattened, having a vertical thickness only $30 \%$ of their radial extent; the inter-density enhancement spacing is comparable to the width of the density enhancement itself. A separate investigation based on high-resolution of the order of $10 \mathrm{~m}$ observations of Saturn's A and B (and probably C) rings should be performed to confirm (or deny!) this our prediction.

We have shown that the self-gravitating rings are able to form various three-dimensional structures - "sausage-like" moderately tightly-wound spirals and rings (Figs. 1b and 3), and clumpy moons ("rubble piles") (Fig. 4), continually forming and dispersing - without the interference of external forces such as embedded or external satellites. Saturn's main rings are likely a dynamic environment, in which the self-gravity Lin-Shu density waves form and dissipate, and gaps and particle clumps form and vanish. Gravitational instability of both ring and spiral perturbations is found to provide an explanation of certain behaviors observed by Tiscareno et al. (2006b, 2008) and Sremčević et al. (2007) for the localized features of Saturn's A ring - "propellers" - which may be interpreted as signatures of 100-m-diameter porous moonlets of preferred mass $M_{\text {moon }} \sim$ $10^{7} \mathrm{~g}$ each embedded within the broad ring. Although this has also yet to be directly measured.
The main shortcoming in our presentation is the short-wave WKB assumption under which all the below considerations are only valid. Without this assumption, Fourier normal modes are no longer solutions of the linearized equations and one has to switch to far more complicated mathematics (e.g., Rüdiger \& Kitchatinov 2000). We intend to address the issue in the following publications of the series. Future work should also include detailed mechanisms of the inelastic interaction: spin degrees of freedom, the particle size distribution, and the finite size of the particles (e.g., Shukhman 1984; Araki 1991). For instance, since the spin of a particle in Saturn's rings is comparable to the orbital frequency, a large amount of energy may be stored in this degree of freedom (e.g., Spilker et al. 2006; Leyrat et al. 2008b). The latter increases the dissipativity of the system, and thus, accelerates the formation of density waves. In local simulations by Salo $(1992,1995)$ and others, one can see spiral wave fragments (cylindric structures of $100 \mathrm{~m}$ or so) with a definite pitch angle with respect to the local shear flow. This angle is almost about $\psi_{\text {crit }}=20^{\circ}$. In Saturn's rings, the preferred orientation of excited gravitationally unstable density waves produces brightness azimuthal variations similar to those observed by Camichel (1958), Lumme \& Irvine (1976), Lumme et al. (1977), Thompson et al. (1981), Franklin et al. (1987), Dones et al. (1993), Dunn et al. (2004), French et al. (2007), and others. Even though the analysis presented here shows that there is a dominant nonaxisymmetric $(\psi \neq 0)$ Fourier mode of maximum instability with $\lambda_{\text {crit }} \approx 100 \mathrm{~m}$, at the present time we cannot explain the angle $\psi_{\text {crit }}$ in the local WKB version of our theory. We also agree with the referee of the paper that in terms of the production of clumpy moonlets this work is highly undeveloped. At present, the paper would have to be regarded as a prediction of moonlet formation by disk instability based on theoretical modeling rather than as an explanation of observed features. Future works should also consider large-scale simulations of Saturn's rings. They could be used both to support the linear dispersion analysis presented here and to examine the nonlinear growth of the instability, especially in terms of the production of porous moonlets.

Acknowledgements. The authors would like to thank Edward Liverts, Yury Lyubarsky, and Michael Mond for stimulating discussions and Irena Zlatopolsky for technical assistance and many comments, which increased the quality of the paper. We are indebted to Alexei M. Fridman for introducing this subject to one of us (E.G.) in 1986 and for his encouragement. We had the pleasure of collaborating with Tzi-Hong Chiueh and Ing-Guey Jiang. This work was sponsored by the Israel Science Foundation and the Binational US - Israel Science Foundation. The efforts of Evgeny Griv for this work were supported in part by the Israeli Ministry of Immigrant Absorption in the framework of the programme "KAMEA".

\section{Appendix A: Stability of a three-dimensional disk}

\section{A.1. Three-dimensional perturbation}

The time-dependent density $\rho(\boldsymbol{r}, t)$, the gravitational potential $\Phi(\boldsymbol{r}, t)$, the pressure $P(\boldsymbol{r}, t)$, and the fluid velocity $\boldsymbol{v}(\boldsymbol{r}, t)$ of a spatially inhomogeneous (only along the $r$ coordinate) disk are represented by

$X(\boldsymbol{r}, t)=X_{0}(z)+X_{1}(\boldsymbol{r}, t)$,

where $X(\boldsymbol{r}, t)$ is any of the above-mentioned physical variables, $X_{0}(z)$ describes the basic flow, and $\left|X_{1} / X_{0}\right| \ll 1$ represents the small perturbations. The stationary $X_{0}$ value of some quantity may of course be equal to zero, as is, for instance, the case for the radial component of the velocity in a dissipationless disk. These quantities $\rho, \Phi, P$, and $v$ are then substituted into the equations 
of motion of the gas, the continuity equation, the Poisson equation, and the second order terms of the order of $\rho_{1}^{2}, \Phi_{1}^{2}, P_{1}^{2}$, and $v^{2}$ - nonlinear terms - are negligible compared to the first order terms. The resultant equations of motion are cyclic in the variables $t$ and $\varphi$, hence by applying the widely used horizontally local WKB method one may determine solutions in the form of normal modes by expanding

$X_{1}(\boldsymbol{r}, t)=\sum_{\boldsymbol{k}} \mathfrak{R}\left\{\tilde{X}_{\boldsymbol{k}}(z) \exp \left[\imath k_{\mathrm{r}}(r) r+\imath m \varphi-\imath \omega_{\boldsymbol{k}} t\right]\right\}$,

where $\tilde{X}_{\boldsymbol{k}}(z)$ is a real amplitude, $m$ is the azimuthal (nonnegative) mode number (=the number of spiral arms), $\omega_{k}=\mathfrak{R} \omega_{k}+l \mathfrak{J} \omega_{k}$ is the complex natural frequency (normal mode) of excited waves, suffixes $\boldsymbol{k}$ denote the $\boldsymbol{k}$ th Fourier component, $t$ is the elapsed time from the onset of the perturbation, $r \sim R,\left|k_{\mathrm{r}}\right| r \gg\left|k_{\mathrm{r}}\right| L \gg 1, R$ is the outer radius of the disk, and $|L|$ is the radial scale of inhomogeneity. The meaning of a localized WKB solution has been discussed in plasma physics (Alexandrov et al. 1984, p. 245; Krall \& Trivelpiece 1986, p. 424; Swanson 1989, p. 142). In general, the WKB approximation can be applied when the medium is weakly inhomogeneous on the scale of the wavelength of the oscillations, i.e., when the wavelength $\lambda=2 \pi / k$ is smaller than the characteristic length $L_{\mathrm{inh}}$ of the inhomogeneity

$\lambda / L_{\text {inh }} \ll 1$.

This is accurate for short wave perturbations only, $\left|k_{\mathrm{r}}\right| r \gg 1$, but qualitatively correct even for perturbations of longer wavelength. The coexistence of several spiral (and ring) waves is possible. In the WKB method,

$k_{\mathrm{r}}^{2} \tilde{X}_{k} \gg \frac{k_{\mathrm{r}}}{r} \tilde{X}_{k} \gg \frac{\tilde{X}_{k}}{r^{2}} \quad$ and $\quad k_{\mathrm{r}} r \gg 1$.

The latter releases us from taking into account the boundary conditions - one indeed considers the disk to be essentially infinite. The radial wavenumber $k_{\mathrm{r}}$ is presumed to be of the form

$k_{\mathrm{r}}(r)=\mathcal{A} \Psi(r)$,

where $\mathcal{A}$ is a large parameter and $\Psi(r)$ is a smooth, slowly and monotonically varying function of the radial distance, i.e., $\mathrm{d} \ln k_{\mathrm{r}} / \mathrm{d} \ln r=O(1)$. It is clearly evident that $X_{1}$ is a periodic function of $\varphi$, hence $m$ must be an integer. In the linear approximation for the lowest local WKB theory, one can select one of the Fourier harmonics

$X_{1}=\mathfrak{R}\left\{\tilde{X}(z) \exp \left(\imath k_{\mathrm{r}} r+\imath m \varphi-\imath \omega t\right)\right\}$,

where now $k_{\mathrm{r}}=$ const. The solution in this form represents in the plane a spiral wave with $m$ arms or a ring $(m=0)$. With $\varphi$ increasing in the rotation direction, we have $k_{\mathrm{r}}>0$ for trailing spiral patterns, which are only observed in Saturn's rings, and $k_{\mathrm{r}}<0$ for leading ones. With $m=0$, we have the density waves in the form of concentric rings that propagate away from the planet when $k_{\mathrm{r}}>0$, or toward the planet when $k_{\mathrm{r}}<0$. The imaginary part of $\omega$ corresponds to a growth $(\mathfrak{J} \omega>0)$ or decay $(\mathfrak{J} \omega<0)$ of the components in time, $X_{1} \propto \exp (\mathfrak{J} \omega t)$, and the real part to a rotation with angular velocity $\Omega_{p}=\mathfrak{R} \omega / m$. When $\mathfrak{J} \omega>0$, the medium transfers its energy to the growing wave and oscillation buildup occurs. A perturbation is considered to be a superposition of different oscillation modes. The disturbance in the disk will grow until it is limited by some nonlinear effect.

In the local WKB approximation, it is assumed that the wave vector and the wavefrequency vary continuously. By utilizing the more accurate nonlocal approximation, it may be shown that the characteristic oscillation frequencies of an inhomogeneous disk must indeed be quantized, i.e., must pass through a discrete series of values (Alexandrov et al. 1984, p. 243). The existence of solutions to linearized gasdynamic equations for a differentially rotating gravitating medium of the form $\exp (-\imath \omega t)$ adopted here was examined by Lominadze et al. (1988) and Fridman (1989). Following Fridman (1989, p. 488), we assume that unstable perturbations develop rapidly on the instability timescale $t_{\text {inst }}$ (see Fridman 1989, for the definition of $t_{\text {inst }}$ ). We refer the interested reader to Lominadze et al. (1988) and Fridman (1989) for details.

\section{A.2. Solutions of the Poisson equation}

Let us consider a disk of particles confined between two boundaries with their unperturbed coordinates, at $z=+h$ and $z=-h$ (Fig. 1a). A disk rotation with the angular velocity vector $\boldsymbol{\Omega}=$ $(0,0, \Omega)$ parallel to the rotational axis $z$ may be assumed. In this configuration, for this Lin-Shu-type perturbation the Poisson equation given by Eq. (4) takes a form

$$
-\left(k_{\mathrm{r}}^{2}+\frac{m^{2}}{r^{2}}\right) \Phi_{1}+\frac{\partial^{2} \Phi_{1}}{\partial z^{2}}=4 \pi G \rho_{1},
$$

where $k_{\mathrm{r}}^{2} \gg m^{2} / r^{2}$. This is the second order approximation in the familiar Lin-Shu asymptotic theory of tightly-wound spirals (Lau \& Bertin 1978; Lin \& Lau 1979; Bertin 1980). The condition $m^{2}\left(k_{\mathrm{r}} r\right)^{-2} \ll 1$ is equivalent to the requirement

$|\tan \psi| \equiv\left|\frac{m}{k_{\mathrm{r}} r}\right| \lesssim 1$,

where $\psi$ is the pitch angle, with " $\lesssim$ " in place of "«" in contrast to the first approximation (Lin \& Shu 1966; Lin et al. 1969; Shu 1970) of the Lin-Shu theory. Equation (A.4) holds for $-h<z<$ $+h$. In the vacuum $(z>+h$ and $z<-h)$, Eq. (A.4) is reduced to the Laplace equation

$-\left(k_{\mathrm{r}}^{2}+\frac{m^{2}}{r^{2}}\right) \Phi_{1}+\frac{\partial^{2} \Phi_{1}}{\partial z^{2}}=0$

therefore, in these regions a solution may be sought that is similar to

$\tilde{\Phi}(z)= \begin{cases}A e^{-k z} & \text { if } z>+h, \\ B e^{+l q z}+C e^{-l q z} & \text { if }-h<z<+h, \\ D e^{+k z} & \text { if } z<-h,\end{cases}$

where $A, B, C$, and $D$ are to be determined and $k=\sqrt{k_{\mathrm{r}}^{2}+(m / r)^{2}}$ is the total planar wave number ${ }^{10}$. To reiterate, this approach has been used in the theory of a bounded plasma confined by a strong magnetic field parallel to the plasma surface (Arsenin 1967; Harris 1968; Alexandrov et al. 1984, p. 313). The constants $A, B, C$, and $D$ are determined by four boundary conditions that both $\Phi_{1}$ and $\partial \Phi_{1} / \partial z$ be continuous at $z=+h$ and $z=-h$

$A \exp (-k h)=B \exp (+l q h)+C \exp (-l q h)$,

$D \exp (-k h)=B \exp (-l q h)+C \exp (+l q h)$,

$k A \exp (-k h)=-\imath q B \exp (+\imath q h)+\imath q C \exp (-\imath q h)$,

$k D \exp (-k h)=+\imath q B \exp (-\imath q h)-\imath q C \exp (+\imath q h)$.

10 This is because for these longitudinal perturbations the equations in Sect. A. 3 below are linear in $z$ (and $r, \varphi, t$ ) and, therefore, we may Fourier-analyze them in the form $\exp (\imath q z) \exp \left(\imath k_{\mathrm{r}} r+\imath m \varphi\right) \exp (-\imath \omega t)$. 
(The potential $\Phi$ must be a continuous function for all $z$ and, in particular, when $z= \pm h$, as otherwise the $z$-component of the force would be infinite.) The condition that this set of equations have a non-trivial solution has the simple form $\left(q^{2}-k^{2}\right) \sin (2 q h)-2 q k \cos (2 q h)=0$, which leads to

$$
\tan (q h)=\frac{k}{q} \quad \text { or } \quad \tan (q h)=-\frac{q}{k} .
$$

Solutions given by Eq. (A.12) is a new result of this work. By using a simplified analysis, very similar equations were already derived by Sekiya (1983, his Eqs. (3.6) and (3.7)), who examined the gravitational instability of the dust layer in the solar nebula. In sharp contrast to our study, however, Sekiya (1983) did not analyze the most important nonaxisymmetric perturbations propagating in a nonuniformly rotating and spatially inhomogeneous disk system.

One can use the first solution of Eqs. (A.12) to describe perturbations that are symmetric with respect to the $z=0$ equatorial plane of the disk (which do not cause it to bend) (Fig. 1b). For these even perturbations with $\Phi_{1}(+z)=\Phi_{1}(-z)$, that is, $A=D$, from Eqs. (A.8) and (A.9) we have $B=C$. The system of of Eqs. (A.8)-(A.11) is then reduced to

$$
A \exp (-k h)=2 B \cos (q h),
$$$$
k A \exp (-k h)=2 q B \sin (q h) \text {, }
$$

from which the required relation, namely $\tan (q h)=k / q$, is easily obtained. This type of vertical motions does not deform the horizontal disk plane $z=0$, because the vertical velocity $v_{z}$ in a density wave is odd in $z$, i.e., $v_{z}(-z)=-v_{z}$, so that in the plane $z=0$ it is equal to zero. The perturbed pressure, density, gravitational potential, and horizontal velocity components are even functions of $z$, while the perpendicular velocity $v_{z}$ is odd in $z$. The even perturbations, forming the basis of the Lin-Shu density wave theory, can release gravitational energy and are subject to Jeans' instability. In other words, we have dealt with the even-parity Jeans perturbations, or compression waves (cf. Chandrasekhar's 1955 singular modes, which have wave vectors perpendicular to the axis of rotation; Goldreich \& Lynden-Bell 1965a), because the perturbed density is an even function of $z$ (see an explanation in Sect. 3.1 above). These perturbations are associated with phenomena such as the appearance of the spiral and ring structures of galaxies (Lin \& Shu 1966; Lin et al. 1969; Yuan 1969; Shu 1970; Griv et al. 1999, 2002, 2006b; Griv 2005b), accretion disks (Alexander et al. 2008), protoplanetary disks (Mayer et al. 2002, 2007; Clampin et al. 2003; Fukagawa et al. 2004, 2006; Boss 2005, 2007, 2008; Rice et al. 2005; Durisen et al. 2007; Griv 2007b; Cossins et al. 2009; Dodson-Robinson et al. 2009), and a protolunar disk (Takeda \& Ida 2001). Examples of even Jeans-unstable, i.e., growing, perturbations are given in both two-dimensional and three-dimensional $N$-body simulations of astrophysical disks (Hohl 1972, 1978; Sellwood \& Athanassoula 1986; Tomley et al. 1991, 1994; Salo 1992, 1995; Richardson 1994; Osterbart \& Willerding 1995; Griv 1998, 2005b, 2007b; Daisaka \& Ida 1999; Ohtsuki \& Emori 2000; Daisaka et al. 2001; Takeda \& Ida 2001; Liverts et al. 2003, Fig. 1 therein; Griv et al. 2006a) and hydrodynamical simulations (Laughlin \& Bodenheimer 1994; Laughlin \& Rózyczka 1996; Gammie 2001; Mayer et al. 2002, 2007; Pickett et al. 2003; Boss 2005, 2007, 2008; Rice et al. 2005; Durisen et al. 2007; Alexander et al. 2008).

After some trigonometric expansions and considering the most important cases of even long-wavelength oscillations (both $|k| h<1$ and $q h<1$ ), from the first solution given by Eq. (A.12) one obtains

$q \approx \sqrt{\frac{k}{h}}$.

The short-wavelength perturbations, $|k| h>1$, are not as dangerous in the problem of system stability as oscillations with $|k| h<1$, since they lead only to very small-scale disturbances of the density with the radial scale $\lambda_{\mathrm{r}}<2 \pi h$. In Saturn's rings, the velocity dispersion of the largest ring particles is $\sqrt{\left\langle v^{2}\right\rangle} \lesssim$ $0.2 \mathrm{~cm} \mathrm{~s}^{-1}$ and the mean epicyclic frequency is $\kappa \approx \Omega \approx \mu \approx 2 \times$ $10^{-4} \mathrm{~s}^{-1}$. Then $h \sim \sqrt{\left\langle v^{2}\right\rangle} / \mu \lesssim 10 \mathrm{~m}$ and $\lambda_{\mathrm{r}}$ is of the order of the largest particle size (Zebker et al. 1985; French \& Nicholson 2000; Esposito 2002). It makes little sense to speak of collective effects on scales smaller than finite-sized particles.

The second solution of Eq. (A.12), $\tan (q h)=-q / k$, describes odd perturbations with $\Phi_{1}(+z)=-\Phi_{1}(-z)$, that is, where $A=-D$ and $B=-C$. This type of vertical motion makes the disk bend in the same way as the plane of an oscillating membrane does (Fig. 1c). The vertical velocity of these motions is an even function of $z: v_{z}(-z)=v_{z}$, and in the plane $z=0$, it is not equal to zero. In contrast to the case of even perturbations just considered above, the perturbed pressure, density, gravitational potential, and horizontal velocity components are odd functions of $z$. These gravity perturbations do not release gravitational energy and, therefore, are expected to be Jeans-stable (Bertin \& Casertano 1982). The bending type of motions can be either caused by tidal influence of a satellite (Shu 1984), or excited by the so-called firehose-type bending instability (e.g., Griv \& Chiueh 1998). Kulsrud et al. (1971) and Mark (1971) investigated the bending instability developing in nonrotating disks by using an energy principle. They demonstrated that the instability is driven by the stellar "pressure" anisotropy: the source of free energy in the instability is the intrinsic planar-to-vertical anisotropy of a velocity dispersion. The firehose instability is well known in plasma physics for transferring energy from one degree of freedom to another (perpendicular) degree of freedom (e.g., Ichimaru 1973). Raha et al. (1991), Griv \& Chiueh (1998), Liverts et al. (2003, Fig. 2 therein), Snytnikov et al. (2004, Fig. 5 therein), and Sotnikova \& Rodionov (2005) presented nonresonant bending-unstable oscillations of three-dimensional rotating $\mathrm{N}$-body models. The study of bending oscillations is beyond the scope of the present paper.

\section{A.3. Dispersion relation}

We next use the momentum equation given by Eq. (1) to determine the perturbed velocity of the fluid element. Based on the assumption that random velocities are anisotropic, the equations of three-dimensional motion of the fluid element (Eq. (1)) in the frame of reference rotating with angular velocity $\Omega$ at the reference position $r_{0}$ can be written in Hill's approximation as (Goldreich \& Lynden-Bell 1965b)

$$
\begin{aligned}
& \frac{\mathrm{d} v_{\mathrm{r}}}{\mathrm{d} t}-2 \Omega v_{\varphi}+2 r r_{1} \Omega \frac{\mathrm{d} \Omega}{\mathrm{d} r}=-\frac{\partial \Phi_{1}^{\prime}}{\partial r}-\frac{4}{3} \eta k_{\mathrm{r}}^{2} v_{\mathrm{r}}, \\
& \frac{\mathrm{d} v_{\varphi}}{\mathrm{d} t}+2 \Omega v_{\mathrm{r}}=-\frac{1}{r} \frac{\partial \Phi_{1}^{\prime}}{\partial \varphi}-\eta k_{\mathrm{r}}^{2} v_{\varphi}, \\
& \frac{\mathrm{d} v_{z}}{\mathrm{~d} t}=-z \mu^{2}-\frac{\partial \Phi_{1}^{\prime}}{\partial z}-\eta k_{\mathrm{r}}^{2} v_{z}
\end{aligned}
$$

where $v_{\mathrm{r}}, v_{\varphi}$, and $v_{z}$ are the radial, azimuthal, and vertical velocities, $r_{1}=\mathrm{d} v_{\mathrm{r}} / \mathrm{d} r, c_{\perp} \sim c_{z} \sim c_{\mathrm{s}}, c_{\mathrm{s}}=(\partial P / \partial \rho)_{0}^{1 / 2}$, 
$\Phi_{1}^{\prime}=\Phi_{1}+c_{\perp}^{2} \rho_{1} / \rho_{0}$, the subscript of $r_{0}$ is dropped as we are considering linearized quantities, $\Omega \equiv \Omega\left(r_{0}\right)$, the influence of the planet enters through $\Omega(r), \eta \approx \xi$, and we use the expansion

$$
P=P_{0}+\rho_{1}(\partial P / \partial \rho)_{0} \quad \text { and } \quad\left|\left(\rho_{1} / P_{0}\right)(\partial P / \partial \rho)_{0}\right| \ll 1 .
$$

In the absence of any perturbing gravity, $\Phi_{1}=\sigma_{1}=\eta=0$, Eqs. (A.14), (A.15) yield the ordinary epicyclic velocities

$$
\begin{aligned}
& v_{\mathrm{r}}=K \cos \left(\phi_{0}-\kappa t\right), \\
& v_{\varphi}=K \frac{2 \Omega}{\kappa} \sin \left(\phi_{0}-\kappa t\right), \\
& v_{z}=N \sin \left(\theta_{0}-\mu t\right),
\end{aligned}
$$

where $K, N, \phi_{0}, \theta_{0}$ are constants of integration, and in Saturn's rings $2 \Omega / \kappa \approx 2$ and $\mu>\Omega>\kappa$ (e.g., Shu 1984). The values of the $r, \varphi$, and $z$ coordinates of the element as functions of time are readily obtained by direct integration of Eqs. (A.17)-(A.19).

Equations (A.14)-(A.16) must be solved simultaneously with the solutions (A.12) and the continuity equation (see Eq. (2))

$$
\begin{aligned}
\rho_{1} & =-\int_{-\infty}^{t}\left[\frac{\partial}{\partial r}\left(\rho_{0} v_{\mathrm{r}}\right)+\frac{1}{r} \frac{\partial}{\partial \varphi}\left(\rho_{0} v_{\varphi}\right)+\frac{\partial}{\partial z}\left(\rho_{0} v_{z}\right)\right] \mathrm{d} t^{\prime} \\
& \approx-\int_{-\infty}^{t}\left(\rho_{0} \frac{\partial v_{\mathrm{r}}}{\partial r}+\frac{\rho_{0}}{r} \frac{\partial v_{\varphi}}{\partial \varphi}+\rho_{0} \frac{\partial v_{z}}{\partial z}\right) \mathrm{d} t^{\prime},
\end{aligned}
$$

where $-\int_{-\infty}^{t} X_{1} \mathrm{~d} t^{\prime}=X_{1}(t) / \iota \omega_{*}, X_{1}(t \rightarrow-\infty)=0$, so by considering only growing perturbations $\left(\mathfrak{J} \omega_{*}>0\right)$ the effects of the initial conditions are neglected, $v_{\mathrm{r}}, v_{\varphi}$, and $v_{z}$ are now the perturbed velocities, $\rho_{0}=\rho_{0}(r, 0)$, and the relatively small term $\rho_{0} v_{\mathrm{r}} / r$ is omitted, i.e., the curvature effect is neglected. This is a valid approximation if $\left|k_{\mathrm{r}}\right| r \gg 1$ (Lau \& Bertin 1978; Lin \& Lau 1979). The particular solution of the system of Eqs. (A.14)-(A.16) is

$v_{\mathrm{r}}=\frac{\left(\omega_{*} k_{\mathrm{r}}+\imath 2 \Omega \frac{m}{r}+\imath \eta k_{\mathrm{r}}^{3}\right) \omega_{*} \boldsymbol{\kappa}}{[\cdots]}$,

$v_{\varphi}=\frac{m}{r} \frac{\aleph}{\omega_{*}}+\frac{\left(\omega_{*} k_{\mathrm{r}}+\imath 2 \Omega \frac{m}{r}+\imath \eta k_{\mathrm{r}}^{3}\right) 2 \Omega \aleph}{\imath[\cdots]}$,

$v_{z}=\frac{\left(\omega_{*} q+\imath \eta k_{\mathrm{r}}^{3}\right) \boldsymbol{\aleph}^{\prime}}{\omega_{*}^{2}-\mu^{2}}$.

In Eqs. (A.21)-(A.23),

$[\cdots]=\left(\omega_{*}^{2}-\kappa^{2}\right) \omega_{*}+\imath \frac{7}{3} \omega_{*}^{2} \eta k_{\mathrm{r}}^{2}-\frac{4}{3} \omega_{*} \eta^{2} k_{\mathrm{r}}^{4}-\imath \eta k_{\mathrm{r}}^{2} 2 r \Omega \frac{\mathrm{d} \Omega}{\mathrm{d} r}$,

$2 r \Omega|\mathrm{d} \Omega / \mathrm{d} r| \sim \Omega,\left|\omega_{*}^{2}\right| \gg \eta^{2} k_{\mathrm{r}}^{4}$,

$\boldsymbol{\aleph}=\left(\delta \Phi+c_{\perp}^{2} \delta \rho / \rho_{0}\right)\left(B e^{+l q z}+B e^{-l q z}\right) \mathrm{e}^{\imath k_{\mathrm{r}} r+l m \varphi-l \omega_{*} t}$,

$\boldsymbol{\aleph}^{\prime}=\left(\delta \Phi+c_{z}^{2} \delta \rho / \rho_{0}\right)\left(B e^{+l q z}-B e^{-l q z}\right) \mathrm{e}^{\imath k_{\mathrm{r}} r+l m \varphi-\imath \omega_{*} t}$,

$\omega_{*}=\omega-m \Omega$ is the Doppler-shifted (in a circular rotating frame) frequency of excited waves, $\omega_{*} \neq 0, \omega_{*}^{2}-\kappa^{2} \neq 0$, and

$\kappa(r)=2 \Omega\left(1+\frac{r}{2 \Omega} \frac{\mathrm{d} \Omega}{\mathrm{d} r}\right)^{1 / 2} \approx \Omega$.

The parameter $\omega_{*}$ is the frequency at which the material, traveling at angular velocity $\Omega$, experience the periodic effect of the spiral pattern of $m$ arms, traveling at angular velocity $\Omega_{\mathrm{p}} \equiv$ $\mathfrak{R} \omega_{*} / m$. One can call $\mathfrak{R} \omega_{*}$ the frequency of encounter of the material with the wave pattern, $\mathfrak{R} \omega_{*} / m$ the rate at which the nonaxisymmetric pattern rotates (the pattern speed), and $\mathfrak{J} \omega_{*}$ the rate of growth. Equations (A.21)-(A.23) describe the small perturbed velocities of the fluid element under the action of the gravity perturbation, $\left|v_{\mathrm{r}}\right|,\left|v_{\varphi}\right|$, and $\left|v_{z}\right| \ll r \Omega$.

A special analysis of the solution close to corotation $\left(\mathfrak{R} \omega_{*} \equiv\right.$ $m\left(\Omega_{\mathrm{p}}-\Omega\right)=0$ ) and Lindblad resonances is required. The Lindblad resonances occur when the mean motion of the wave and that of the disk particles are in the ratio

$$
\frac{\Omega-\Omega_{\mathrm{p}}}{\kappa}= \pm \frac{1}{m}
$$

and in Saturn's rings $\kappa \approx \Omega$. The most important are strong loworder resonances with $m<10$ (see Goldreich \& Tremaine 1982; Shu 1984; and Griv 2007b, for an explanation). When conditions given by Eq. (A.24) hold for the upper (lower) choice of sign, we refer to $r_{\text {ILR }}$ and $r_{\mathrm{OLR}}$ as the inner (outer) Lindblad horizontal resonance, respectively.

The set of Eqs. (A.20)-(A.23) is a system of algebraic equations. Using Eqs. (A.13) and (A.21)-(A.23), from Eqs. (A.4) and (A.20), it is straightforward to show that (e.g., Griv 2006)

$$
\begin{aligned}
\omega_{*}^{3} & +\omega_{*}^{2} l \frac{7}{3} \eta k_{\mathrm{r}}^{2}-\omega_{*} \omega_{\mathrm{J}}^{2}-\imath\left[2 r \Omega \frac{\mathrm{d} \Omega}{\mathrm{d} r}\right. \\
& \left.-\frac{2 \pi G \sigma_{0}|k|-k^{2} c_{\perp}^{2}(1+|k| h)}{1+|k| h}\right] \eta k_{\mathrm{r}}^{2}=0,
\end{aligned}
$$

where

$$
\begin{aligned}
\omega_{\mathrm{J}}^{2}= & \kappa^{2}-\frac{2 \pi G \sigma_{0}|k|-k^{2} c_{\perp}^{2}(1+|k| h)}{1+|k| h} \\
& \times\left(1+\frac{m^{2}}{r^{2} k^{2}} \frac{4 \Omega^{2}-\kappa^{2}+\omega_{*}^{2}}{\omega_{*}^{2}}\right)
\end{aligned}
$$

is the square of the Jeans frequency and $m^{2} / r^{2} k^{2} \ll 1,|k| h \lesssim 1$. The algebraic equation Eq. (A.25) is the Lin-Shu-type dispersion relation for the density waves developing in a thin threedimensional disk, that is, the relationship between the frequency of excited oscillations $\omega_{*}$ and the wave vector $\boldsymbol{k}$.

Our dispersion relation given by Eq. (A.25) reduces to those of Lau \& Bertin (1978), Lin \& Lau (1979), Morozov (1985), Montenegro et al. (1999), and Griv et al. (2002) when the finite thickness, "out-of-phase", and resonant contributions vanish, so it seems as correct as their result. Equation (A.25) is complicated: it is highly nonlinear in the frequency $\omega_{*}$. To deal with the most interesting oscillation types analytically, we consider various limiting cases of perturbations described by some simplified variations in the dispersion relation. We first restrict ourselves to considerating the principal part of the system between the inner and outer Lindblad resonances, $\left|\omega_{*}^{2}\right| \ll \kappa^{2}$, and in particular, to the transparency region between the turning points $\left(\omega_{*}= \pm \kappa\right)$ in a disk. In the opposite case, $\left|\omega_{*}\right|>\kappa$, the effect of the disk rotation is negligible and therefore irrelevant to us. Therefore, this limit never applies to the rapidly rotating subsystems of both spiral galaxies and planetary rings. Second, we can solve Eq. (A.25) by successive approximations. In the low-frequency $\left(\left|\omega_{*}^{2}\right|<\kappa^{2}\right)$ and local WKB approximations $\left(k_{\mathrm{r}}^{2} \gg m^{2} / r^{2}\right)$ that we indeed explore in Eq. (A.25), the terms that describe tangential forces $\propto m$ are assumed to be small compared to other terms and, therefore, may be neglected to the lowest approximation. In addition, the viscous effects are also considered to be weak, $\kappa \sim \Omega \sim \mu \gg \eta k_{\mathrm{r}}^{2}$. 


\section{A.4. Gravitational instability}

From Eq. (A.25) for the most important high-frequency range in which we are interested

$\left|\omega_{*}\right| \sim\left|\omega_{\mathrm{J}}\right| \sim \Omega \gg \eta k_{\mathrm{r}}^{2}$,

we determine the dispersion law for the Jeans branch of oscillations. By neglecting the small terms $\propto m^{2} / r^{2}, h$, and all dissipative effects $\propto \eta$, one obtains, in the lowest approximation, the familiar Lin-Shu dispersion relation for axisymmetric $\left(\tan \psi \equiv m / k_{\mathrm{r}} r=0\right)$ waves

$\omega_{*}^{2}=\kappa^{2}-2 \pi G \sigma_{0}\left|k_{\mathrm{r}}\right|+k_{\mathrm{r}}^{2} c_{\mathrm{s}}^{2}$

(Lin et al. 1969). Accordingly, the disk is Jeans' gravitationally unstable $\left(\omega_{*}^{2}<0\right)$ to axisymmetric perturbations if

$c_{\mathrm{s}}<c_{\mathrm{T}}=\frac{\pi G \sigma_{0}}{\kappa}$

where $c_{\mathrm{T}}$ is the ordinary Safronov-Toomre (Safronov 1960, 1980; Toomre 1964) critical sound speed to suppress the instability of only axisymmetric (or radial) perturbations.

In the next approximation, in Eqs. (A.25) and (A.28), considering the Safronov-Toomre stable disk $\left(c_{\mathrm{S}} \geq c_{\mathrm{T}}\right)$ and the most important low-frequency oscillations $\left(\left|\omega_{*}^{2}\right| \lesssim \kappa^{2}\right)$, in the small terms $\propto \mathrm{m}^{2} / r^{2}$ one can replace $\omega_{*}^{2}$ by $\kappa^{2}$. As a result, the dispersion relation is obtained in the form

$$
\begin{gathered}
\omega_{*}^{3}+\omega_{*}^{2} l \frac{7}{3} \eta k_{\mathrm{r}}^{2}-\omega_{*} \omega_{\mathrm{J}}^{2}-\imath\left(2 r \Omega \frac{\mathrm{d} \Omega}{\mathrm{d} r}\right. \\
\left.-\frac{2 \pi G \sigma_{0}|k|-k^{2} c_{\mathrm{s}}^{2}}{1+|k| h}\right) \eta k_{\mathrm{r}}^{2}=0,
\end{gathered}
$$

where $\left|\omega_{*}\right| \lesssim \kappa,|k| h \lesssim 1$,

$$
\omega_{\mathrm{J}}^{2} \approx \kappa^{2}-\frac{2 \pi G \sigma_{0}\left(k_{*}^{2} /|k|\right)}{1+|k| h}+k_{*}^{2} c_{\mathrm{s}}^{2}
$$

is now the square of the Jeans frequency,

$k_{*}^{2}=k^{2}\left\{1+\left[(2 \Omega / \kappa)^{2}-1\right] \sin ^{2} \psi\right\}$

is the squared effective wavenumber, and in Saturn's rings $(2 \Omega / \kappa)^{2}-1 \approx 3$. Equation (A.30) differs from the ordinary Lin-Shu dispersion relation (A.28) by the appearance of the total $k$ and effective $k_{*}$ wavenumbers, which originate from the consideration of the nonaxisymmetrical modes $\propto \psi$, by the factor $\propto h$, which originates from the consideration of the effects of the finite thickness, and by the factor $\propto \eta$, which originates from the consideration of the viscous effects. Lynden-Bell \& Kalnajs (1972, their Eq. (A11)) first obtained the Lin-Shu-type dispersion relation for open $(\psi \neq 0)$ waves propagating in a homogeneous, dissipationless disk. A simplified dispersion relation (A.30) for low-frequency $\left|\omega_{*}\right|^{2}<\kappa^{2}$ perturbations that we are interested in can easily be obtain from Eqs. (D12) (in Eq. (D12) it should be $T_{1} /\left(1-v^{2}\right)$ instead of $T_{1}$; Montenegro et al. 1989) and (D14) of Lin \& Lau (1979) by ignoring the viscous effects and the "out-of-phase" term $\imath k_{\mathrm{r}} A$, and using the expansion $2 \Omega / \kappa \approx 1-(r / 4 \Omega)(\mathrm{d} \Omega / \mathrm{d} r)$.

From Eq. (A.30), we determine the dispersion law for the Jeans branch of oscillations

$\omega_{* 1,2} \approx \pm p\left|\omega_{\mathrm{J}}\right|-\imath \frac{7}{6} \eta k_{\mathrm{r}}^{2}$ where $p=1$ for gravity-stable perturbations with $\omega_{*}^{2} \approx \omega_{\mathrm{J}}^{2}>0$, $p=l$ for gravity-unstable perturbations with $\omega_{*}^{2} \approx \omega_{\mathrm{J}}^{2}<$ 0 , and the term involving $\eta$ is the small correction $\left(\left|\omega_{\mathrm{J}}\right| \gg\right.$ $\eta k_{\mathrm{r}}^{2}$ ). Equation (A.32) determines the spectrum of oscillations. Accordingly, viscosity leads to a weak damping of both Jeansunstable and Jeans-stable density waves. Thus, even though the damping rate of the instability due to collisions $(\eta \neq 0)$ is not constant (as the density increases in the nonlinear part of the instability, e.g., Daisaka et al. 2001), this does not modify the papers' conclusions.

It follows from Eq. (A.32) that the growth rate of the instability is high, $\mathfrak{J} \omega_{* 1,2} \approx \sqrt{2 \pi G \sigma_{0}\left(k_{*}^{2} / k\right)}$, and in general

$\mathfrak{J} \omega_{* 1,2} \sim \Omega$

that is, the instability develops rapidly on a dynamical timescale.

Equation (A.31) indicates the stabilizing influence of a finite thickness. Use of the dispersion curve minimum condition, $\partial \omega_{*}^{2} / \partial k=0$, in Eq. (A.31) determines the wavelength of the most unstable Jeans mode

$\lambda_{\text {crit }} \approx \frac{2 c_{\mathrm{s}}^{2}}{G \sigma_{0}}\left(1+\frac{2 \pi G \sigma_{0} h}{c_{\mathrm{s}}^{2}}\right) \approx 4 \pi h$,

where $2 \pi G \sigma_{0} h / c_{\mathrm{s}}^{2}<1$, and only wavelengths close to $\lambda_{\text {crit }}$ are unstable. As one can see from the above equation, the finite thickness $(h \neq 0)$ shifts the threshold of instability toward a longer wavelength. Use of the condition $\omega_{*}^{2} \geq 0$ determines the marginal sound speed for the stability of arbitrary but not only axisymmetric perturbations developing in a rapidly rotating, three-dimensional, and spatially homogeneous disk

$c_{\mathrm{s}} \gtrsim c_{\text {crit }} \approx \frac{2 \Omega}{\kappa} c_{\mathrm{T}}\left(1-\frac{\kappa h}{2 c_{\mathrm{T}}}\right)$,

where $2 \Omega / \kappa \approx 2$ and $\kappa h / 2 c_{\mathrm{T}}<1$. The inequality in Eq. (A.34) defines the modified Safronov-Toomre stabilization criterion of Jeans' gravitational instability in a real differentially rotating $(2 \Omega / \kappa>1)$ and three-dimensional $(h \neq 0)$ system. The longitudinal (along the flow velocity) size of the structure of the gravitationally unstable disk is determined from Eq. (A.33). When we consider the axisymmetric two-dimensional case, i.e., $\psi=0^{\circ}$ and $h=0$, then because $k_{\phi} \equiv m / r=0$ (see Eq. (A.26)), our result reduces to the familiar Safronov-Toomre criterion $c_{\mathrm{T}}$. The observations of Saturn's rings reflect the general cases, not $m=0$. Therefore, they should agree more closely with our general result given by Eq. (A.34), which is indeed the case.

From Eqs. (A.29) and (A.34), it follows that once a disk is unstable according to the above criterions, namely $c_{\perp}<$ $c_{\mathrm{T}}$, both axisymmetric modes and nonaxisymmetric modes of comparable wavelengths, should increase at comparable rates; the disk should break up in patches. It also follows that the viscosity has no important effect on the criterion of the gravitational instability. Equation (A.34) improves the SafronovToomre stability criterion $c_{\mathrm{T}}$ by including destabilizing effects resulting from shear $\propto 2 \Omega / \kappa \propto|\mathrm{d} \Omega / \mathrm{d} r|$ and azimuthal forces $\propto m$ (Griv et al. 2002; Griv 2006), and a stabilizing effect resulting from finite thickness $\propto h$. Maxwell (1859) considered just this kind of spiral instabilities with $m=1$ in his study concerning the stability of the Saturnian uniform rings whose radial extent was considerably larger than the average interparticle distance. Maxwell assumed that, in such a system, the azimuthal force resulting from azimuthal displacements was more important in determining the stability than the radial 
force produced by radial displacements. In particular, Goldreich \& Lynden-Bell (1965a, p. 123) noted that "... in the galaxy the tangential modes may be most unstable". Lau \& Bertin (1978, p. 509) (see also Bertin 1980; Morozov 1980) then clarified the problem by considering the motion of a fluid element: the density response that is in phase with the potential minimum is found to exceed, by an amount proportional to both $|\mathrm{d} \Omega / \mathrm{d} r|$ and $m$, the corresponding response due to an axisymmetric field of equal strength. In Toomre (1981), this amplification was discussed in terms of a "swing mechanism", very reminiscent of the way we reach the modified stability criterion (A.34). (The swing-amplified mechanism was discovered by Goldreich \& Lynden-Bell 1965b. The swing works on leading waves and turns them into trailing waves producing strong amplification in the process.) The free kinetic energy associated with the differential rotation of the system is one possible source for the growth of the energy of these spiral gravity perturbations, and appears to be released when angular momentum is transferred outward.

Vandervoort (1970), Yue (1982), Shu (1984), Morozov (1981), Romeo (1992, 1994), and Osterbart \& Willerding (1995) already obtained somewhat similar stabilizing factors $\propto h$ by using simplified theories.

\section{A.5. Dissipative instability}

In contrast to Eq. (A.27), in the frequency range

$\left|\omega_{*}\right| \sim \eta k_{\mathrm{r}}^{2} \ll\left|\omega_{\mathrm{J}}\right|$,

Eq. (A.25) has another root equal to

$\omega_{* 3} \approx l\left(\frac{2 \pi G \sigma_{0}|k|-k^{2} c_{\perp}^{2}}{1+|k| h}-2 r \Omega \frac{\mathrm{d} \Omega}{\mathrm{d} r}\right) \frac{\eta k_{\mathrm{r}}^{2}}{\omega_{\mathrm{J}}^{2}}$,

where $\mathrm{d} \Omega / \mathrm{d} r \approx-(3 / 2)(\Omega / r), \omega_{\mathrm{J}}^{2} \sim \Omega^{2}>0$, and $\left|\omega_{* 3}^{2} / \omega_{\mathrm{J}}^{2}\right| \ll 1$. Equation (A.36) indicates the same condition for disk instability (for which $\mathfrak{J} \omega_{* 3}>0$ ) as in the usual hydrodynamic description adopted by Lynden-Bell \& Pringle (1974), Mishurov et al. (1976), and Morozov et al. (1985): a viscous gravitationally stable $\left(\omega_{\mathrm{J}}^{2}>0\right)$ disk is aperiodically $\left(\mathfrak{R} \omega_{* 3}=0\right.$ and $\left.\mathfrak{J} \omega_{* 3}>0\right)$ unstable (in a rotating frame) for modes with wavelength

$\lambda>\lambda_{\mathrm{J}}=\frac{c_{\perp}^{2}}{G \sigma_{0}}$.

As seen, the instability develops even in a rigidly rotating system. Because the limit $\mathfrak{J} \omega_{* 3} / \Omega \ll 1$ is considered, the instability develops on the timescale of many disk rotations. Equation (A.37) is just the Jeans criterion for hydrodynamic instability in a flat medium without rotation, and $\lambda_{\mathrm{J}}$ is the well known Jeans critical wavelength that separates unstable (at $\lambda>$ $\left.\lambda_{\mathrm{J}}\right)$ and stable $\left(\lambda<\lambda_{\mathrm{J}}\right)$ perturbations. The viscosity thus removes the rotational stabilization in a flat system. This was first found by Lynden-Bell \& Pringle (1974).

Following Lynden-Bell \& Pringle (1974), Mishurov et al. (1976), and Morozov et al. (1985), we also call the instability the dissipative instability because it is introduced by collisions between particles (and the disk's self-gravity). In our analysis, we make the basic physical assumption that the transport coefficients $\eta$ and $\xi$ are independent of state variables, in particular of the density $\rho$. We have just shown above that these viscousunstable perturbations grow aperiodically $\left(\mathfrak{R} \omega_{* 3}=0\right)$. We note that the instability has nothing to do with the viscous instability (viscous overstability) discussed by Borderies et al. (1985) and Schmit \& Tscharnuter (1995).
In general, the effect of viscosity is to disrupt the organized wave motion. A wave therefore tends to be damped on a timescale of $1 / v_{\text {coll }}$ (Bohm \& Gross 1949). Lynden-Bell \& Pringle (1974), however, demonstrated that not all the organized motion will be lost in a self-gravitating system: a dissipative type instability can develop even in a Jeans-stable, rigidly rotating self-gravitating viscous disk. According to Morozov et al. (1985), the introduction of differential rotation leaves the result of Lynden-Bell \& Pringle (1974) unchanged. Morozov et al. (1985) also obtained the important result that the growth rate of the dissipative instability reaches a maximum in the part of the disk that is marginally Jeans-stable gravitationally. We refer to Willerding (1992) for the effect of simultaneous action of viscosity and self-gravity on rotating disks.

The cause of the instability, which has an essential dependence on the self-gravitation of the disk matter, was explained in Lynden-Bell \& Pringle (1974), Mishurov et al. (1976), and Morozov et al. (1985). Lynden-Bell \& Pringle claimed this instability to be analogous to the well-known viscous mechanism that converts Maclaurin spheroids to Jacobi ellipsoids. The dissipative instability, as well as the Jeans one studied above, can be considered as generating mechanisms for unstable short-scale density waves, and these waves might be responsible for the appearance of structures in galaxies, Saturn's rings, and protoplanetary disks (Morozov et al. 1985; Gorkavyi \& Fridman 1990a,b). In the framework of our theory, however, the Saturnian ring disk is gravitationally unstable $\left(\omega_{\mathrm{J}}^{2}<0\right)$ and therefore the weak $\left(\mathfrak{J} \omega_{* 3} / \Omega \ll 1\right)$ dissipative instability in Saturn's rings studied in this section is unlikely. We find evidence that the growth rate of the dissipative instability is indeed small in the case of low and relatively high optical depth rings making Jeans' gravitational instability studied in Sect. A.4 above a leading candidate mechanism to explain both axisymmetric and nonaxisymmetric microstructures observed in Saturn's A and B rings (and probably in its $\mathrm{C}$ ring as well).

\section{References}

Alexander, R. D., Armitage, P. J., Cuadra, J., \& Begelman, M. C. 2008, ApJ, 674,927

Alexandrov, A. F., Bogdankevich, L. S., \& Rukhadze, A. A. 1984, Principles of Plasma Electrodynamics (New York: Springer)

Altobelli, N., Spilker, L. J., Leyrat, C., \& Pilorz, S. 2008, Planet. Space Sci., 56, 134

Araki, S. 1991, Am. Sci., 79, 44

Arsenin, V. V. 1967, Soviet Phys. - Tech. Phys., 12, 442

Bertin, G. 1980, Phys. Rep., 61, 1

Bertin, G. 2000, Dynamics of Galaxies (Cambridge: Cambridge Univ. Press)

Bertin, G., \& Casertano, S. 1982, A\&A, 106, 274

Bertin, G., \& Lin, C. C. 1996, Spiral Structure in Galaxies: A Density Wave Theory (Cambridge: MIT Press)

Bohm, D., \& Gross, E. P. 1949, Phys. Rev., 75, 1864

Borderies, N., \& Longaretti, P.-Y. 1994, Icarus, 107, 129

Borderies, N., Goldreich, P., \& Tremaine, S. 1985, Icarus, 63, 406

Boss, A. 2005, ApJ, 629, 535

Boss, A. 2007, ApJ, 661, L73

Boss, A. 2008, ApJ, 677, 607

Brahic, A. 2001, in Granular Gases, ed. T. Pöschel, \& A. Luding (Berlin: Springer), 281

Bridges, F. G., Hatzes, A. P., \& Lin, D. N. C. 1984, Nature, 309, 333

Brown, R. H., Baines, K. H., Bellucci, G., et al. 2006, A\&A, 446, 707

Camichel, H. 1958, Ann. d'Astr., 21, 231

Chagelishvili, G. D., Zahn, J.-P., Tevzadze, A. G., \& Lominadze, J. G. 2003, A\&A, 402, 401

Chandrasekhar, S. 1955, Vistas Astron., 1, 344

Clampin, M., Krist, J. E., Ardila, D. R., et al. 2003, AJ, 126, 385 Colombo, G., Goldreich, P., \& Harris, A. 1976, Nature, 264, 344 Colwell, J. E., Esposito, L. W., \& Sremčević, M. 2006, Geophys. Res. Lett., 33, L07201 
Colwell, J. E., Esposito, L. W., Sremčević, M., Stewart, G. R., \& McClintock, W. E. 2007, Icarus, 90, 127

Colwell, J. E., Cooney, J. H., Esposito, L. W., \& Sremčević, M. 2009, Icarus, 200,574

Cook, A. F., \& Franklin, F. A. 1964, AJ, 69, 17

Cossins, P., Lodato, G., \& Clarke, C. J. 2009, MNRAS, 393, 1157

Cuzzi, J. N., Colwell, J. E., Esposito, L. W., et al. 2002, Space Sci. Rev., 118, 209

Cuzzi, J., Clark, R., Filacchione, G., et al. 2009, in Saturn from Cassini-Huygens, ed. M. K. Dougherty, L. W. Esposito, \& S. M. Krimigis (Netherlands: Springer), 459

Daisaka, H., \& Ida, S. 1999, Earth Planets Space, 51, 1195

Daisaka, H., Tanaka, H., \& Ida, S. 2001, Icarus, 154, 296

Dodson-Robinson, S. E., Veras, D., Ford, E. B., \& Beichman, C. A. 2009, ApJ, 707, 79

Dones, L., Cuzzi, J. N., \& Showalter, M. R. 1993, Icarus, 105, 184

Dunn, D. E., Molnar, L. A., Niehof, J. T., de Pater, I., \& Lissauer, J. J. 2004, Icarus, 171,183

Durisen, R. H., Boss, A. P., Mayer, L., et al. 2007, in Protostars and Planets V, ed. B. Reipurth, D. Jewitt, \& K. Keil (Tucson: Univ. Arizona Press), 607

Esposito, L. W. 2002, Rep. Prog. Phys., 65, 1741

Esposito, L. W., O'Callaghan, M., \& West, R. A. 1983, Icarus, 56, 439

Ferrari, C., Galdemard, P., Lagage, P. O., Pantin, E., \& Quoirin, C. 2005, A\&A, 441,379

Ferrari, C., Brooks, S., Edgington, S., et al. 2009, Icarus, 199, 145

Franklin, F. A., \& Colombo, G. 1978, Icarus, 33, 279

Franklin, F. A., Cook, A. F., Barrey, R. T. F., et al. 1987, Icarus, 69, 280

French, R. G., \& Nicholson, P. D. 2000, Icarus, 145, 502

French, R. G., Salo, H., McGhee, C. A., \& Dones, L. 2007, Icarus, 189, 493

Fridman, A. M. 1989, Soviet Ast. Lett., 15, 487

Fukagawa, M., Hayashi, M., Tamura, M., et al. 2004, ApJ, 605, L53

Fukagawa, M., Tamura, M., Itoh, Y., et al. 2006, ApJ, 636, L153

Galeev, A. A., \& Sagdeev, R. Z. 1983, in Basic Plasma Physics, ed. M. N.

Rosenbluth, \& R. Z. Sagdeev (Amsterdam: North-Holland), 1, 679

Gammie, C. F. 2001, ApJ, 553, 174

Ginzburg, I. F., Polyachenko, V. L., \& Fridman, A. M. 1972, Soviet Ast., 15, 643

Goldreich, P., \& Lynden-Bell, D. 1965a, MNRAS, 130, 97

Goldreich, P., \& Lynden-Bell, D. 1965b, MNRAS, 130, 125

Goldreich, P., \& Tremaine, S. 1982, ARA\&A, 20, 249

Gorkavyi, N. N., \& Fridman, A. M. 1990a, Soviet Phys. - Uspehi, 33, 95

Gorkavyi, N. N., \& Fridman, A. M. 1990b, Soviet Ast. Lett., 16, 79

Griv, E. 1998, Planet. Space Sci., 46, 615

Griv, E. 2005a, in Dynamics of Populations of Planetary Systems, ed. Z. Knežević, \& A. Milani (Cambridge: Cambridge Univ. Press), IAU Colloq., 197,459

Griv, E. 2005b, Ap\&SS, 299, 371

Griv, E. 2006, A\&A, 449, 573

Griv, E. 2007a, ApJ, 665, 866

Griv, E. 2007b, Planet. Space Sci., 55, 547

Griv, E., \& Chiueh, T. 1998, ApJ, 503, 186

Griv, E., \& Gedalin, M. 2003, Planet. Space Sci., 51, 899

Griv, E., \& Gedalin, M. 2004, AJ, 128, 1956

Griv, E., \& Gedalin, M. 2005, Planet. Space Sci., 53, 461

Griv, E., \& Gedalin, M. 2006, Planet. Space Sci., 54, 794

Griv, E., Rosenstein, B., Gedalin, M., \& Eichler, D. 1999, A\&A, 347, 821

Griv, E., Gedalin, M., Eichler, D., \& Yuan, C. 2000, Planet. Space Sci., 48, 679

Griv, E., Gedalin, M., \& Yuan, C. 2002, A\&A, 383, 338

Griv, E., Gedalin, M., \& Yuan, C. 2003a, A\&A, 400, 375

Griv, E., Gedalin, M., \& Yuan, C. 2003b, MNRAS, 342, 1102

Griv, E., Gedalin, M., \& Lyubarsky, Yu. 2006a, Adv. Space Res., 38, 770

Griv, E., Gedalin, M., \& Yuan, C. 2006b, Adv. Space Res., 38, 47

Griv, E., Liverts, E., \& Mond, M. 2008, A\&A, 672, L127

Harris, E. G. 1968, in Physics of Hot Plasmas, ed. B. J. Rye, \& J. C. Taylor

(New York: Plenum), 145

Hedman, M. M., Nicholson, P. D., Salo, H., et al. 2007, AJ, 133, 2624

Hedman, M. M., Burns, J. A., Tiscareno, M. S., \& Porco, C. C. 2009, Planet. Space Sci., 202, 260

Hohl, F. 1972, J. Comput. Phys., 9, 10

Hohl, F. 1978, AJ, 83, 768

Holberg, J. B., Forrester, W. T., \& Lissauer, J. J. 1982, Nature, 297, 115

Horne, L. J., \& Cuzzi, J. N. 1996, Icarus, 119, 285

Ichimaru, S. 1973, Basic Principles of Plasma Physics (Reading: Benjamin)

Jeans, J. H. 1928, Astronomy and Cosmogony (Cambridge: Cambridge Univ. Press)

Jeffreys, H. 1947, MNRAS, 107, 263

Julian, W. H., \& Toomre, A. 1966, ApJ, 146, 810

Karjalainen, R., \& Salo, H. 2004, Icarus, 172, 328

Karttunen, H. 1983, Moon Planets, 29, 117
Kerr, R. A. 1985, Science, 220, 1376

Krall, N. A., \& Trivelpiece, A. W. 1986, Principles of Plasma Physics (San Francisco: San Francisco Press)

Kulsrud, R. M. 1972, in Gravitational N-body Problem, ed. M. Lecar (Dordrecht: Reidel), 337

Kulsrud, R. M., Mark, J. W.-K., \& Caruso, A. 1971, Ap\&SS, 14, 52

Landau, L. D., \& Lifshitz, E. M. 1987, Fluid Mechanics (Oxford: ButtenworthHeinemann)

Lane, A. L., Hord, C. W., West, R. A., et al. 1982, Science, 215, 537

Lau, Y. Y., \& Bertin, G. 1978, ApJ, 226, 508

Laughlin, G., \& Bodenheimer, P. 1994, ApJ, 436, 335

Laughlin, G., \& Rózyczka, M. 1996, ApJ, 456, 279

Leyrat, C., Spilker, L. J., Altobelli, N., Pilorz, S., \& Ferrari, C. 2008a, Planet. Space Sci., 56, 117

Leyrat, C., Ferrari, C., Charnoz, S., et al. 2008b, Icarus, 196, 625

Lin, C. C., \& Shu, F. H. 1966, Proc. Natl. Acad. Sci., 55, 229

Lin, C. C., \& Shu, F. H. 1968, in Astrophysics and General Relativity, ed. M. Chretian, S. Deser, \& J. Goldstein (New York: Gordon and Breach), 2, 236

Lin, C. C., \& Lau, Y. Y. 1979, SIAM Stud. Appl. Math., 60, 97

Lin, D. N. C., \& Paploizou, J. C. B. 1996, ARA\&A, 34, 703

Lin, C. C., Yuan, C., \& Shu, F. H. 1969, ApJ, 155, 721 (erratum, 156, 797)

Lissauer, J. J., \& Cuzzi, J. N. 1982, Icarus, 87, 1051

Liverts, E., Griv, E., Gedalin, M., \& Eichler, D. 2003, in Galaxies and Chaos, ed. G. Contopoulos, \& N. Voglis (Berlin: Springer), 340

Lominadze, D. G., Chagelishvili, G. D., \& Chanishvili, R. G. 1988, Soviet Ast. Lett., 14, 364

Lumme, K., \& Irvine, W. M. 1976, ApJ, 204, L55

Lumme, K., \& Irvine, W. M. 1979, Nature, 282, 695

Lumme, K., Esposito, L. W., Irvine, W. M., \& Baum, W. A. 1977, ApJ, 216, L123

Lynden-Bell, D., \& Kalnajs, A. J. 1972, MNRAS, 157, 1

Lynden-Bell, D., \& Pringle, J. E. 1974, MNRAS, 168, 603

Maoz, E. 1995, ApJ, 455, L131

Mark, J. W.-K. 1971, ApJ, 169, 455

Mayer, L., Quinn, T., Wadsley, J., \& Stadel, J. 2002, Science, 298, 1756

Mayer, L., Lufkin, G., Quinn, T., \& Wadsley, J. 2007, ApJ, 661, L77

Maxwell, J. C. 1859, in Sci. Papers, 1960, ed. W. D. Niven (New York: Dover), 1,28

Mishurov, Yu. N., Peftiev, V. N., \& Suchkov, A. A. 1976, Soviet Ast., 20, 152

Montenegro, L. E., Yuan, C., \& Elmegreen, B. G. 1999, ApJ, 520, 592

Morozov, A. G. 1980, Soviet Ast., 24, 391

Morozov, A. G. 1981, Soviet Ast. Lett., 7, 109

Morozov, A. G. 1985, Soviet Ast., 29, 120

Morozov, A. G., Torgashin, Yu. M., \& Fridman, A. M. 1985, Soviet Ast. Lett., 11,94

Mosqueira, I. 1996, Icarus, 122, 128

Murray, C. D., Beurle, K., Cooper, N. J., et al. 2008, Nature, 453, 739

Nicholson, P. D., \& Dones, L. 1991, Rev. Geophys. Suppl., 29, 313

Nicholson, P. D., \& Hedman, M. M. 2010, Icarus, 206, 410

Nicholson, P. D., French, R. G., Tollestrup, E., et al. 2000, Icarus, 145, 474

Nicholson, P. D., French, R. G., Campbell, D. B., et al. 2005, Icarus, 177, 32

Nicholson, P. D., Hedman, M. M., Clark, R. N., et al. 2008, Icarus, 193, 182

Ohtsuki, K., \& Emori, H. 2000, AJ, 119, 403

Osterbart, R., \& Willerding, E. 1995, Planet. Space Sci., 43, 289

Papaloizou, J. C. B., \& Lin, D. N. C. 1995, ARA\&A, 33, 505

Porco, C. C. 1990, Adv. Space Res., 10, 221

Porco, C. C., Baker, E., Barbara, J., et al. 2005, Science, 307, 1226

Porco, C. C., Weiss, J. W., Richardson, D. C., et al. 2008, AJ, 136, 2172

Postnikov, E. B., \& Loskutov, A. Yu. 2007, J. Exper. Theor. Phys., 104, 417

Pickett, B. K., Mejía, A. C., Durisen, R. H., et al. 2003, ApJ, 590, 1060

Raha, N., Sellwood, J. A., James, R. A., \& Kahn, F. D. 1991, Nature, 352, 411

Rice, W. K. M., Lodato, G., \& Armitage, P. J. 2005, MNRAS, 364, L56

Richardson, D. C. 1994, MNRAS, 269, 493

Romeo, A. B. 1992, MNRAS, 256, 307

Romeo, A. B. 1994, A\&A, 286, 799

Rosen, P. A., Tyler, G. L., Marouf, E. A., \& Lissauer, J. J. 1991, Icarus, 93, 25

Rüdiger, G., \& Kitchatinov, L. L. 2000, Astron. Nachr., 21, 181

Safronov, V. S. 1960, Ann. Astrn., 23, 979

Safronov, V. S. 1980, in Early Solar System Processes, ed. D. Lal (Amsterdam: North-Holland), 73

Sagdeev, R. Z., \& Galeev, A. A. 1969, Nonlinear Plasma Theory, ed. T. M. O’Neil, \& D. L. Book (New York: Benjamin)

Salo, H. 1992, Nature, 359, 619

Salo, H. 1995, Icarus, 117, 287

Schmit, U., \& Tscharnuter, W. M. 1995, Icarus, 115, 304

Sekiya, M. 1983, Prog. Theor. Phys., 69, 1116

Sellwood, J. A., \& Athanassoula, E. 1986, MNRAS, 221, 195

Sellwood, J. A., \& Kahn, F. D. 1991, MNRAS, 250, 278 
Sellwood, J. A., \& Lin, D. C. 1989, MNRAS, 240, 991

Showalter, M. R., \& Nicholson, P. D. 1990, Icarus, 87, 285

Shu, F. H. 1970, ApJ, 160, 99

Shu, F. H. 1984, in Planetary Rings, ed. R. Greenberg, \& A. Brahic (Tucson: Univ. Arizona Press), 513

Shu, F. H., \& Stewart, G. R. 1985, Icarus, 62, 360

Shu, F. H., Cuzzi, J. N., \& Lissauer, J. J. 1983, Icarus, 53, 185

Shu, F. H., Dones, L., Lissauer, J. J., Yuan, C., \& Cuzzi, J. N. 1985, ApJ, 299, 542

Shukhman, I. G. 1984, Soviet Ast., 28, 574

Sicardy, B. 2005, Space Sci. Rev., 116, 457

Smith, B. A., Soderblom, L., Beebe, R. F., et al. 1981, Science, 212, 163

Smith, B. A., Soderblom, R., Batson, R., et al. 1982, Science, 215, 504

Snytnikov, V. N., Vshivkov, V. A., Kuksheva, E. A., et al. 2004, Astr. Lett., 30, 124

Sotnikova, N. Ya., \& Rodionov, S. A. 2005, Astr. Lett., 31, 15

Spilker, L. J., Pilorz, S. H., Wallis, B. D., et al. 2006, Planet. Space Sci., 54, 1167

Sremčević, M., Schmidt, J., Salo, H., et al. 2007, Nature, 449, 1019

Stewart, G., Lin, D. N. C., \& Bodenheimer, P. 1984, in Planetary Rings, ed. R.

Greenberg, \& A. Brahic (Tucson: Univ. Arizona Press), 447

Stone, E. C., \& Miner, E. D. 1982, Science, 215, 499

Swanson, D. G. 1989, Plasma Waves (Boston: Academic Press)

Takeda, T., \& Ida, S. 2001, ApJ, 560, 514
Thompson, W. T., Lumme, K., Irvine, W. M., Baum, W. A., \& Esposito, L. W. 1981, Icarus, 46, 187

Thomson, F. S., Marouf, E. A., Tyler, G. L., French, R. G., \& Rappoport, N. J. 2007, Geophys. Res. Lett., 34, L24203

Tiscareno, M. S., Nicholson, P. D., Burns, J. A., Hedman, M. M., \& Porco, C. C. 2006a, ApJ, 651, L65

Tiscareno, M. S., Burns, J. A., Hedman, M. M., et al. 2006b, Nature, 440, 648

Tiscareno, M. S., Burns, J. A., Nicholson, P. D., Hedman, M. M., \& Porco, C. C. 2007, Icarus, 189, 14

Tiscareno, M. S., Burns, J. A., Hedman, M. M., \& Porco, C. C. 2008, AJ, 135, 1083

Tiscareno, M. S., Perrine, R. P., Richardson, D. C., et al. 2010, AJ, 139, 492

Tomley, L., Cassen, P., \& Steiman-Cameron, T. 1991, ApJ, 382, 530

Tomley, L., Steiman-Cameron, T., \& Cassen, P. 1994, ApJ, 422, 850

Toomre, A. 1964, ApJ, 139, 1217

Toomre, A. 1981, in The Structure and Evolution of Normal Galaxies, ed. S. F.

Fall, \& D. Lynden-Bell (Cambridge: Cambridge Univ. Press), 111

Vandervoort, P. O. 1970, ApJ, 161, 87

Willerding, E. 1992, Earth Moon Planets, 56, 173

Yuan, C. 1969, ApJ, 158, 871

Yuan, C. 2002, Space Sci. Rev., 102, 121

Yue, Z. Y. 1982, Geophys. Astrophys. Fluid Dyn., 20, 1

Zebker, H. A., Marouf, E. A., \& Tyler, G. L. 1985, Icarus, 84, 531 